\title{
On the Cauchy problem for the new integrable two-component Novikov equation
}

\author{
Yongsheng $\mathrm{Mi}^{1} \cdot$ Chunlai $\mathrm{Mu}^{2}$
}

Received: 15 June 2019 / Accepted: 14 September 2019 / Published online: 28 November 2019 (c) Fondazione Annali di Matematica Pura ed Applicata and Springer-Verlag GmbH Germany, part of Springer Nature 2019

\begin{abstract}
This paper is devoted to a new integrable two-component Novikov equation, lax pairs and biHamiltonian structures. Firstly, the local well-posedness in nonhomogeneous Besov spaces is established by using the Littlewood-Paley theory and transport equations theory. Then, we verify the blow-up that occurs for this system only in the form of breaking waves. Moreover, with analytic initial data, we show that its solutions are analytic in both variables, globally in space and locally in time. Finally, we prove that the strong solutions of the system maintain corresponding properties at infinity within its lifespan provided the initial data decay exponentially and algebraically, respectively.
\end{abstract}

Keywords Besov spaces · Camassa-Holm-type equation · Local well-posedness · Persistence properties

Mathematics Subject Classification 35B30 $\cdot 35 \mathrm{G} 25 \cdot 35 \mathrm{~A} 10 \cdot 35 \mathrm{Q} 53$

\section{Introduction}

In this paper, we consider the following Cauchy problem of the two-component Novikov equation

$$
\begin{cases}m_{t}+2 u v m_{x}+2 u v_{x} m+4 v u_{x} m=0, & t>0, x \in \mathbb{R}, \\ n_{t}+2 u v n_{x}+2 v u_{x} n+4 u v_{x} n=0, & t>0, x \in \mathbb{R}, \\ m=u-u_{x x}, n=v-v_{x x}, & t>0, x \in \mathbb{R}, \\ u(0, x)=u_{0}(x), v(0, t)=v_{0}(x), & x \in \mathbb{R}\end{cases}
$$

$凶 \quad$ Yongsheng Mi

miyongshen@163.com

1 College of Mathematics and Statistics, Yangtze Normal University, Chongqing 408100, People's Republic of China

2 College of Mathematics and Statistics, Chongqing University, Chongqing 401331, People’s Republic of China 
The two-component Novikov equation (1.1) was found by Li [46]. It was shown in [46] that the system (1.1) appears in the bi-Hamiltonian form

$$
\left(\begin{array}{l}
u \\
v
\end{array}\right)_{t}=\Phi(m, n)\left(\begin{array}{l}
\frac{\delta H}{\delta m} \\
\frac{\delta H}{\delta n}
\end{array}\right)=\Psi(m, n)\left(\begin{array}{l}
\frac{\delta W}{\delta m} \\
\frac{\delta W}{\delta n}
\end{array}\right),
$$

with Hamiltonian pair

$$
\begin{aligned}
\Phi(m, n)= & \frac{1}{2}\left(\begin{array}{c}
3 m \partial+2 m_{x} \\
3 n \partial+2 n_{x}
\end{array}\right)\left(\partial^{3}-4 \partial\right)^{-1}\left(3 m \partial+m_{x} 3 n \partial+n_{x}\right) \\
& +\frac{1}{2}\left(\begin{array}{c}
m \partial^{-1} m-m \partial^{-1} n \\
-n \partial^{-1} m n \partial^{-1} n
\end{array}\right), \\
\Psi(m, n)= & -\frac{1}{4}\left(\partial^{2}-1\right)\left(\begin{array}{cc}
m \partial^{-1} n & m \partial^{-1} n+n \partial^{-1} m \\
m \partial^{-1} n+n \partial^{-1} m & m \partial^{-1} m
\end{array}\right)\left(\partial^{2}-1\right) \\
= & -\frac{1}{12}\left(\partial^{2}-1\right)\left(\begin{array}{cc}
-\frac{1}{m} \partial^{\frac{m}{n}} \partial^{-1} \frac{1}{m} & \frac{1}{m}\left(2-\left(\frac{m}{n}\right)_{x} \partial^{-1} \frac{n}{m}\right) \partial \frac{1}{n} \\
\frac{1}{n}\left(2-\left(\frac{v}{m}\right)_{x} \partial^{-1} \frac{m}{n}\right) \partial \frac{1}{m} & -\frac{1}{n} \partial \frac{n}{m} \partial^{-1} \frac{1}{n}
\end{array}\right)\left(\partial^{2}-1\right) .
\end{aligned}
$$

The associated Hamiltonian functional is $H=\int m v+n u \mathrm{~d} x$, and $W$ is nonlocal and looks very complicated, so we omit it.

In recent decades, the Camassa-Holm $(\mathrm{CH})$-type equations raised a lot of interest because of their specific properties, one of which is that they possess peakon solutions (peaked soliton solutions with discontinuous derivatives at the peaks). The most celebrated member of them is the Camassa-Holm equation [17]

$$
u_{t}-u_{t x x}+3 u u_{x}=2 u_{x} u_{x x}+u u_{x x x}
$$

modeling the unidirectional propagation of shallow water waves over a flat bottom, $u(t, x)$ stands for the fluid velocity at time $t$ in the spatial direction $x$. It is a well-known integrable equation describing the velocity dynamics of shallow water waves. This equation spontaneously exhibits emergence of singular solutions from smooth initial conditions. It has a bi-Hamilton structure [24] and is completely integrable [7,17]. In particular, it possesses an infinity of conservation laws and is solvable by its corresponding inverse scattering transform. After the birth of the Camassa-Holm equation, many works have been carried out to probe its dynamic properties. For example, Eq. (1.4) has traveling wave solutions of the form $c e^{-|x-c t|}$, called peakons, which describes an essential feature of the traveling waves of largest amplitude (see $[8,11,12]$ ). It is shown in $[9,13,16]$ that the inverse spectral or scattering approach is a powerful tool to handle the Camassa-Holm equation and analyze its dynamics. It is worthwhile to mention that Eq. (1.4) gives rise to geodesic flow of a certain invariant metric on the Bott-Virasoro group [14,51], and this geometric illustration leads to a proof that the Least Action Principle holds. It is shown in [10] that the blow-up occurs in the form of breaking waves, namely the solution remains bounded but its slope becomes unbounded in finite time. Moreover, the Camassa-Holm equation has global conservative solutions [4,37] and dissipative solutions $[3,36]$.

In 1999, Degasperis and Procesi used an asymptotic integrability approach to isolate integrable third-order equations, and discovered a new $\mathrm{CH}$-type equation, i.e., the DegasperisProcesi (DP) equation [22]

$$
u_{t}-u_{t x x}+4 u u_{x}=3 u_{x} u_{x x}+u u_{x x x} .
$$

Degasperis, Holm and Hone [22] proved the formal integrability of Eq. (1.5) by constructing a Lax pair. They also showed that it has bi-Hamiltonian structure and an infinite sequence 
of conserved quantities and admits exact peakon solutions. The direct and inverse scattering approach to pursue it can be seen in [18]. Moreover, in [23], they also presented that the Degasperis-Procesi equation has a bi-Hamiltonian structure and an infinite number of conservation laws, and admits exact peakon solutions which are analogous to the Camassa-Holm peakons. It is worth pointing out that solutions of this type are not mere abstractizations: The peakons replicate a feature that is characteristic for the waves of great height-waves of largest amplitude that are exact solutions of the governing equations for irrotational water waves cf. the papers $[8,11,57]$. The Degasperis-Procesi equation is a model for nonlinear shallow water dynamics cf. the discussion in [15]. The numerical stability of solitons and peakons, the multisoliton solutions and their peakon limits, together with an inverse scattering method to compute $N$-peakon solutions to Degasperis-Procesi equation have been investigated, respectively, in $[38,49,50]$. Furthermore, the traveling wave solutions and the classification of all weak traveling wave solutions to Degasperis-Procesi equation were presented in $[45,58]$.

Both the $\mathrm{CH}$ and DP equations are the third-order $\mathrm{CH}$-type equations with quadratic nonlinearity. Recently, by the symmetry classification study of nonlocal partial differential equations with quadratic or cubic nonlinearity, Novikov discovered a new $\mathrm{CH}$-type equation with cubic nonlinearity [54]

$$
u_{t}-u_{t x x}+4 u^{2} u_{x}=3 u u_{x} u_{x x x}+u^{2} u_{x x x}
$$

which has been recently discovered by Vladimir Novikov in a symmetry classification of nonlocal PDEs with quadratic or cubic nonlinearity [54]. The perturbative symmetry approach yields necessary conditions for a PDE to admit infinitely many symmetries. Using this approach, Novikov was able to isolate Eq. (1.6) and find its first few symmetries, and he subsequently found a scalar Lax pair for it, then proved that the equation is integrable, which can be thought as a generalization of the Camassa-Holm equation. In [39], it is shown that the Novikov equation admits peakon solutions like the Camassa-Holm. Also, it has a Lax pair in matrix form and a bi-Hamiltonian structure. Furthermore, it has infinitely many conserved quantities. Like Camassa-Holm, the most important quantity conserved by a solution $u$ to Novikov equation is its $H^{1}$-norm $\|u\|_{H^{1}}^{2}=\int_{R}\left(u^{2}+u_{x}^{2}\right)$, which plays an important role in the study of Eq. (1.6). In [32,52,59]; the authors study well-posedness and dependence on initial data for the Cauchy problem for Novikov equation. Recently, in [42], a global existence result and conditions on the initial data were considered. Existence and uniqueness of global weak solution to Novikov equation with initial data under some conditions were proved in [60]. The Novikov equation with dissipative term was considered in [64]. Multipeakon solutions were studied in $[39,40]$. The Cauchy problem of the Novikov equation on the circle was investigated in [56]. The two-component Novikov equation may be reduced to the Novikov equation (1.6) under the constraint $u=v$.

Later Geng and Xue [25] constructed the following two-component Novikov equation

$$
\left\{\begin{array}{l}
m_{t}+3 u_{x} v m+u v m_{x}=0, \\
n_{t}+3 v_{x} u m_{x}+u u_{x} n_{x}=0, \\
m=u-u_{x x}, n=v-v_{x x},
\end{array}\right.
$$

which was associated with a $3 \times 3$ matrix spectral problem, they also gave the $N$ peakons, infinite sequence of conserved quantities and a Hamiltonian structure. In 2013, Li and Liu showed the system (1.7) was indeed a bi-Hamiltonian structure and got the Hamiltonian operators found by Hone and Wang for the Novikov equation (1.6) using the proper Dirac reduction [48]. In [28], it is shown that an Novikov system (1.7) is well-posed in Sobolev spaces $H^{s}$ for $s>3 / 2$, in the sense of Hadamard. Furthermore, it is proved that the dependence on initial data is sharp, i.e., the data-to-solution map is continuous but not uniformly 
continuous. Also, peakon traveling wave solutions are used to prove that the solution map is not uniformly continuous in $H^{s}$ for $s<3 / 2$.

It is worthwhile to note that there is also research on other multi-component $\mathrm{CH}$-type equations [41,61-63].

To our best knowledge, the Cauchy problem (1.1) has not been studied yet. In this paper, firstly, the local well-posedness of the Cauchy problem (1.1) in nonhomogeneous Besov spaces $B_{p, r}^{s} \times B_{p, r}^{s}$ with $p, r \in[1, \infty], s>\max \left\{\frac{5}{2}, 2+\frac{1}{p}\right\}$ is established by using the Littlewood-Paley theory and transport equations theory. Then, we verify the blow-up that occurs for this system only in the form of breaking waves. Moreover, with analytic initial data, we show that its solutions are analytic in both variables, globally in space and locally in time. Finally, we prove that the strong solutions of the system maintain corresponding properties at infinity within its lifespan provided the initial data decay exponentially and algebraically, respectively.

The rest of this paper is organized as follows. In Sect. 2, we prove the local well-posedness of the initial-value problem (1.1). Section 3 supplies a blow-up criteria. Section 4 is devoted to the study of the analyticity of the Cauchy problem (1.1). In Sect. 5, two persistence properties for the strong solutions are given.

\section{Local well-posedness in the Besov spaces}

In this section, we shall establish local well-posedness of the initial-value problem (1.1) in the Besov spaces.

First, for the convenience of the readers, we recall some facts on the Littlewood-Paley decomposition and some useful lemmas.

Notation $\mathcal{S}$ stands for the Schwartz space of smooth functions over $\mathbb{R}^{d}$ whose derivatives of all order decay at infinity. The set $\mathcal{S}^{\prime}$ of temperate distributions is the dual set of $\mathcal{S}$ for the usual pairing. We denote the norm of the Lebesgue space $L^{p}(\mathbb{R})$ by $\|\cdot\|_{L^{p}}$ with $1 \leq p \leq \infty$, and the norm in the Sobolev space $H^{s}(\mathbb{R})$ with $s \in \mathbb{R}$ by $\|\cdot\|_{H^{s}}$.

Proposition 2.1 (Littlewood-Paley decomposition [5]) Let $\mathcal{B} \doteq\left\{\xi \in \mathbb{R}^{d},|\xi| \leq \frac{4}{3}\right\}$ and $\mathcal{C} \doteq\left\{\xi \in \mathbb{R}^{d}, \frac{4}{3} \leq|\xi| \leq \frac{8}{3}\right\}$. There exist two radial functions $\chi \in C_{c}^{\infty}(\mathcal{B})$ and $\varphi \in C_{c}^{\infty}(\mathcal{C})$ such that

$$
\begin{aligned}
& \chi(\xi)+\sum_{q \geq 0} \varphi\left(2^{-q} \xi\right)=1, \forall \xi \in \mathbb{R}^{d}, \\
& \left|q-q^{\prime}\right| \geq 2 \Rightarrow \operatorname{Supp\varphi }\left(2^{-q} \cdot\right) \cap \operatorname{Supp} \varphi\left(2^{-q^{\prime}} \cdot\right)=\varnothing, \\
& q \geq 1 \Rightarrow \operatorname{Supp} \chi(\cdot) \cap \operatorname{Supp} \varphi\left(2^{-q^{\prime}} \cdot\right)=\varnothing, \\
& \frac{1}{3} \leq \chi(\xi)^{2}+\sum_{q \geq 0} \varphi\left(2^{-q} \xi\right)^{2} \leq 1, \forall \xi \in \mathbb{R}^{d} .
\end{aligned}
$$

Furthermore, let $h \doteq \mathcal{F}^{-1} \varphi$ and $\tilde{h} \doteq \mathcal{F}^{-1} \chi$. Then for all $f \in \mathcal{S}^{\prime}\left(\mathbb{R}^{d}\right)$, the dyadic operators $\Delta_{q}$ and $S_{q}$ can be defined as follows

$$
\begin{aligned}
\Delta_{q} f \doteq \varphi\left(2^{-q} D\right) f=2^{q d} \int_{\mathbb{R}^{d}} h\left(2^{q} y\right) f(x-y) \mathrm{d} y \text { for } q \geq 0, \\
S_{q} f \doteq \chi\left(2^{-q} D\right) f=\sum_{-1 \leq k \leq q-1} \Delta_{k}=2^{q d} \int_{\mathbb{R}^{d}} \tilde{h}\left(2^{q} y\right) f(x-y) \mathrm{d} y,
\end{aligned}
$$




$$
\Delta_{-1} f \doteq S_{0} f \text { and } \Delta_{q} f \doteq 0 \text { for } q \leq-2
$$

Hence,

$$
f=\sum_{q \geq 0} \Delta_{q} f \text { in } \mathcal{S}^{\prime}\left(\mathbb{R}^{d}\right),
$$

where the right-hand side is called the nonhomogeneous Littlewood-Paley decomposition of $f$.

Lemma 2.1 (Bernstein's inequality [21]) Let $\mathcal{B}$ be a ball with center 0 in $\mathbb{R}^{d}$ and $\mathcal{C}$ a ring with center 0 in $\mathbb{R}^{d}$. A constant $C$ exists so that, for any positive real number $\lambda$, any nonnegative integer $k$, any smooth homogeneous function $\sigma$ of degree $m$ and any couple of real numbers $(a, b)$ with $b \geq a \geq 1$, there hold

$$
\begin{aligned}
& \text { Supp } \hat{u} \subset \lambda \mathcal{B} \Rightarrow \sup _{|\alpha|=k}\left\|\partial^{\alpha} u\right\|_{L^{a}} \leq C^{k+1} \lambda^{k+d\left(\frac{1}{a}-\frac{1}{b}\right)}\|u\|_{L^{a}}, \\
& \text { Suppû } \subset \lambda \mathcal{C} \Rightarrow C^{-k-1} \lambda^{k}\|u\|_{L^{a}} \leq \sup _{|\alpha|=k}\left\|\partial^{\alpha} u\right\|_{L^{a}} \leq C^{k+1} \lambda^{k}\|u\|_{L^{a}}, \\
& \text { Suppî } \subset \lambda \mathcal{C} \Rightarrow\|\sigma(D) u\|_{L^{b}} \leq C_{\sigma, m} \lambda^{m+d\left(\frac{1}{a}-\frac{1}{b}\right)}\|u\|_{L^{a}},
\end{aligned}
$$

for any function $u \in L^{a}$.

Definition 2.1 (Besov space) Let $s \in \mathbb{R}, 1 \leq p, r \leq \infty$. The inhomogeneous Besov space $B_{p, r}^{s}\left(\mathbb{R}^{d}\right)\left(B_{p, r}^{s}\right.$ for short) is defined by

$$
B_{p, r}^{s} \doteq\left\{f \in \mathcal{S}^{\prime}\left(\mathbb{R}^{d}\right) ;\|f\|_{B_{p, r}^{s}}<\infty\right\}
$$

where

$$
\|f\|_{B_{p, r}^{s}} \doteq \begin{cases}\left(\sum_{q \in \mathbb{Z}} 2^{q s r}\left\|\Delta_{q} f\right\|_{L_{p}}^{r}\right)^{\frac{1}{r}}, & \text { for } r<\infty, \\ \sup _{q \in \mathbb{Z}} 2^{q s}\left\|\Delta_{q} f\right\|_{L_{p}}, & \text { for } r=\infty .\end{cases}
$$

If $s=\infty, B_{p, r}^{\infty} \doteq \cap_{s \in \mathbb{R}} B_{p, r}^{s}$.

Proposition 2.2 (see [21]) Suppose that $s \in \mathbb{R}, 1 \leq p, r, p_{i}, r_{i} \leq \infty(i=1,2)$. We have

(1) Topological properties: $B_{p, r}^{s}$ is a Banach space which is continuously embedded in $\mathcal{S}^{\prime}$.

(2) Density: $C_{c}^{\infty}$ is dense in $B_{p, r}^{s} \Leftrightarrow 1 \leq p, r \leq \infty$.

(3) Embedding: $\left.B_{p_{1}, r_{1}}^{s} \hookrightarrow B_{p_{2}, r_{2}}^{s-n\left(\frac{1}{p_{1}}-\frac{1}{p_{2}}\right.}\right)$, if $p_{1} \leq p_{2}$ and $r_{1} \leq r_{2} . B_{p, r_{2}}^{s_{2}} \hookrightarrow B_{p, r_{1}}^{s_{1}}$ locally compact, if $s_{1}<s_{2}$.

(4) Algebraic properties: $\forall s>0, B_{p, r}^{s} \cap L^{\infty}$ is an algebra. Moreover, $B_{p, r}^{s}$ is an algebra, provided that $s>\frac{n}{p}$ or $s \geq \frac{n}{p}$ and $r=1$.

(5) Complex interpolation:

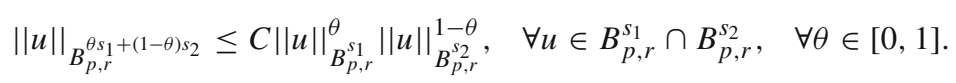

(6) Fatou lemma: If $\left(u_{n}\right)_{n \in \mathbb{N}}$ is bounded in $B_{p, r}^{s}$ and $u_{n} \rightarrow u$ in $\mathcal{S}^{\prime}$, then $u \in B_{p, r}^{s}$ and

$$
\|u\|_{B_{p, r}^{s}} \leq \liminf _{n \rightarrow \infty}\left\|u_{n}\right\|_{B_{p, r}^{s}} .
$$


(7) Let $m \in \mathbb{R}$ and $f$ be an $S^{m}$-multiplier (i.e., $f: \mathbb{R}^{d} \rightarrow \mathbb{R}$ is smooth and satisfies that $\forall \alpha \in \mathbb{N}^{d}$, there exists a constant $C_{\alpha}$, s.t. $\left|\partial^{\alpha} f(\xi)\right| \leq C_{\alpha}\left(1+|\xi|^{m-|\alpha|}\right)$ for all $\left.\xi \in \mathbb{R}^{d}\right)$. Then the operator $f(D)$ is continuous from $B_{p, r}^{s}$ to $B_{p, r}^{s-m}$.

Now we state some useful results in the transport equation theory, which are crucial to the proofs of our main theorems later.

Proposition 2.1. (Littlewood-Paley decomposition [5]) Let $\mathcal{B} \doteq\left\{\xi \in \mathbb{R}^{d},|\xi| \leq \frac{4}{3}\right\}$ and $\mathcal{C} \doteq\left\{\xi \in \mathbb{R}^{d}, \frac{4}{3} \leq|\xi| \leq \frac{8}{3}\right\}$. There exist two radial functions $\chi \in C_{c}^{\infty}(\mathcal{B})$ and $\varphi \in C_{c}^{\infty}(\mathcal{C})$ such that

$$
\begin{aligned}
& \chi(\xi)+\sum_{q \geq 0} \varphi\left(2^{-q} \xi\right)=1, \forall \xi \in \mathbb{R}^{d}, \\
& \left|q-q^{\prime}\right| \geq 2 \Rightarrow \operatorname{Supp} \varphi\left(2^{-q} \cdot\right) \cap \operatorname{Supp} \varphi\left(2^{-q^{\prime}} \cdot\right)=\varnothing, \\
& q \geq 1 \Rightarrow \operatorname{Supp} \chi(\cdot) \cap \operatorname{Supp} \varphi\left(2^{-q^{\prime}} \cdot\right)=\varnothing, \\
& \frac{1}{3} \leq \chi(\xi)^{2}+\sum_{q \geq 0} \varphi\left(2^{-q} \xi\right)^{2} \leq 1, \forall \xi \in \mathbb{R}^{d} .
\end{aligned}
$$

Furthermore, let $h \doteq \mathcal{F}^{-1} \varphi$ and $\tilde{h} \doteq \mathcal{F}^{-1} \chi$. Then for all $f \in \mathcal{S}^{\prime}\left(\mathbb{R}^{d}\right)$, the dyadic operators $\Delta_{q}$ and $S_{q}$ can be defined as follows

$$
\begin{aligned}
& \Delta_{q} f \doteq \varphi\left(2^{-q} D\right) f=2^{q d} \int_{\mathbb{R}^{d}} h\left(2^{q} y\right) f(x-y) \mathrm{d} y \text { for } q \geq 0 \\
& S_{q} f \doteq \chi\left(2^{-q} D\right) f=\sum_{-1 \leq k \leq q-1} \Delta_{k}=2^{q d} \int_{\mathbb{R}^{d}} \tilde{h}\left(2^{q} y\right) f(x-y) \mathrm{d} y, \\
& \Delta_{-1} f \doteq S_{0} f \text { and } \Delta_{q} f \doteq 0 \text { for } q \leq-2 .
\end{aligned}
$$

Hence,

$$
f=\sum_{q \geq 0} \Delta_{q} f \text { in } \mathcal{S}^{\prime}\left(\mathbb{R}^{d}\right),
$$

where the right-hand side is called the nonhomogeneous Littlewood-Paley decomposition of $f$.

Lemma 2.1.(Bernstein's inequality [21]) Let $\mathcal{B}$ be a ball with center 0 in $\mathbb{R}^{d}$ and $\mathcal{C}$ a ring with center 0 in $\mathbb{R}^{d}$. A constant $C$ exists so that, for any positive real number $\lambda$, any nonnegative integer $k$, any smooth homogeneous function $\sigma$ of degree $m$ and any couple of real numbers $(a, b)$ with $b \geq a \geq 1$, there hold

$$
\begin{aligned}
& \text { Supp } \hat{u} \subset \lambda \mathcal{B} \Rightarrow \sup _{|\alpha|=k}\left\|\partial^{\alpha} u\right\|_{L^{a}} \leq C^{k+1} \lambda^{k+d\left(\frac{1}{a}-\frac{1}{b}\right)}\|u\|_{L^{a}}, \\
& \text { Supp } \hat{u} \subset \lambda \mathcal{C} \Rightarrow C^{-k-1} \lambda^{k}\|u\|_{L^{a}} \leq \sup _{|\alpha|=k}\left\|\partial^{\alpha} u\right\|_{L^{a}} \leq C^{k+1} \lambda^{k}\|u\|_{L^{a}}, \\
& \text { Supp } \hat{u} \subset \lambda \mathcal{C} \Rightarrow\|\sigma(D) u\|_{L^{b}} \leq C_{\sigma, m} \lambda^{m+d\left(\frac{1}{a}-\frac{1}{b}\right)}\|u\|_{L^{a}},
\end{aligned}
$$

for any function $u \in L^{a}$.

Definition 2.1. (Besov space) Let $s \in \mathbb{R}, 1 \leq p, r \leq \infty$. The inhomogeneous Besov space $B_{p, r}^{s}\left(\mathbb{R}^{d}\right)\left(B_{p, r}^{s}\right.$ for short) is defined by

$$
B_{p, r}^{s} \doteq\left\{f \in \mathcal{S}^{\prime}\left(\mathbb{R}^{d}\right) ;\|f\|_{B_{p, r}^{s}}<\infty\right\}
$$


where

$$
\|f\|_{B_{p, r}^{s}} \doteq \begin{cases}\left(\sum_{q \in \mathbb{Z}} 2^{q s r}\left\|\Delta_{q} f\right\|_{L_{p}}^{r}\right)^{\frac{1}{r}}, & \text { for } r<\infty, \\ \sup _{q \in \mathbb{Z}} 2^{q s}\left\|\Delta_{q} f\right\|_{L_{p}}, & \text { for } r=\infty .\end{cases}
$$

If $s=\infty, B_{p, r}^{\infty} \doteq \cap_{s \in \mathbb{R}} B_{p, r}^{s}$.

Proposition 2.2. (see [21]) Suppose that $s \in \mathbb{R}, 1 \leq p, r, p_{i}, r_{i} \leq \infty(i=1,2)$. We have

(1) Topological properties: $B_{p, r}^{s}$ is a Banach space which is continuously embedded in $\mathcal{S}^{\prime}$.

(2) Density: $C_{c}^{\infty}$ is dense in $B_{p, r}^{s} \Leftrightarrow 1 \leq p, r<\infty$.

(3) Embedding: $B_{p_{1}, r_{1}}^{s} \hookrightarrow B_{p_{2}, r_{2}}^{s-n\left(\frac{1}{p_{1}}-\frac{1}{p_{2}}\right)}$, if $p_{1} \leq p_{2}$ and $r_{1} \leq r_{2} . B_{p, r_{2}}^{s_{2}} \hookrightarrow B_{p, r_{1}}^{s_{1}}$ locally compact, if $s_{1}<s_{2}$.

(4) Algebraic properties: $\forall s>0, B_{p, r}^{s} \cap L^{\infty}$ is an algebra. Moreover, $B_{p, r}^{s}$ is an algebra, provided that $s>\frac{n}{p}$ or $s \geq \frac{n}{p}$ and $r=1$.

(5) Complex interpolation:

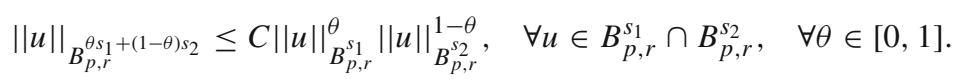

(6) Fatou lemma: If $\left(u_{n}\right)_{n \in \mathbb{N}}$ is bounded in $B_{p, r}^{s}$ and $u_{n} \rightarrow u$ in $\mathcal{S}^{\prime}$, then $u \in B_{p, r}^{s}$ and

$$
\|u\|_{B_{p, r}^{s}} \leq \liminf _{n \rightarrow \infty}\left\|u_{n}\right\|_{B_{p, r}^{s}} .
$$

(7) Let $m \in \mathbb{R}$ and f be an $S^{m}$-multiplier (i.e., $f: \mathbb{R}^{d} \rightarrow \mathbb{R}$ is smooth and satisfies that $\forall \alpha \in \mathbb{N}^{d}$, there exists a constant $C_{\alpha}$, s.t. $\left|\partial^{\alpha} f(\xi)\right| \leq C_{\alpha}\left(1+|\xi|^{m-|\alpha|}\right)$ for all $\left.\xi \in \mathbb{R}^{d}\right)$. Then the operator $f(D)$ is continuous from $B_{p, r}^{s}$ to $B_{p, r}^{s-m}$.

Now we state some useful results in the transport equation theory, which are crucial to the proofs of our main theorems later.

Lemma 2.2 (see [20,21]) Suppose that $(p, r) \in[1,+\infty]^{2}$ and $s>-\frac{d}{p}$. Let $v$ be a vector field such that $\nabla v$ belongs to $L^{1}\left([0, T] ; B_{p, r}^{s-1}\right)$ if $s>1+\frac{d}{p}$ or to $L^{1}\left([0, T] ; B_{p, r}^{\frac{d}{p}} \cap L^{\infty}\right)$ otherwise. Suppose also that $f_{0} \in B_{p, r}^{s}, F \in L^{1}\left([0, T] ; B_{p, r}^{s}\right)$ and that $f \in L^{\infty}\left(L^{1}\left([0, T] ; B_{p, r}^{s}\right) \cap\right.$ $C\left([0, T] ; \mathcal{S}^{\prime}\right)$ solves the $d$-dimensional linear transport equations

$$
\text { (T) }\left\{\begin{array}{l}
\partial_{t} f+v \cdot \nabla f=F \\
\left.f\right|_{t=0}=f_{0}
\end{array}\right.
$$

Then there exists a constant $C$ depending only on $s, p$ and $d$ such that the following statements hold:

(1) If $r=1$ or $s \neq 1+\frac{d}{p}$, then

$$
\|f\|_{B_{p, r}^{s}} \leq\left\|f_{0}\right\|_{B_{p, r}^{s}}+\int_{0}^{t}\|F(\tau)\|_{B_{p, r}^{s}} \mathrm{~d} \tau+C \int_{0}^{t} V^{\prime}(\tau)\|f(\tau)\|_{B_{p, r}^{s}} \mathrm{~d} \tau,
$$

or

$$
\|f\|_{B_{p, r}^{s}} \leq e^{C V(t)}\left(\left\|f_{0}\right\|_{B_{p, r}^{s}}+\int_{0}^{t} e^{-C V(\tau)}\|F(\tau)\|_{B_{p, r}^{s}} \mathrm{~d} \tau\right)
$$

hold, where $V(t)=\int_{0}^{t}\|\nabla v(\tau)\|_{B_{p, r}^{\frac{d}{p}} \cap L^{\infty}} \mathrm{d} \tau$ ifs $<1+\frac{d}{p}$ and $V(t)=\int_{0}^{t}\|\nabla v(\tau)\|_{B_{p, r}^{s-1}} \mathrm{~d} \tau$ else. 
(2) If $s \leq 1+\frac{d}{p}$ and $\nabla f_{0} \in L^{\infty}, \nabla f \in L^{\infty}\left([0, T] \times \mathbb{R}^{d}\right)$ and $\nabla F \in L^{1}\left([0, T]\right.$; $\left.L^{\infty}\right)$, then $\|f\|_{B_{p, r}^{s}}+\|\nabla f\|_{L^{\infty}}$

$$
\leq e^{C V(t)}\left(\left\|f_{0}\right\|_{B_{p, r}^{s}}+\left\|\nabla f_{0}\right\|_{L^{\infty}}+\int_{0}^{t} e^{-C V(\tau)}\left(\|F(\tau)\|_{B_{p, r}^{s}}+\|\nabla F(\tau)\|_{L^{\infty}}\right) \mathrm{d} \tau\right)
$$

with $V(t)=\int_{0}^{t}\|\nabla v(\tau)\|_{B_{p, r}^{\frac{d}{p}} \cap L^{\infty}} \mathrm{d} \tau$.

(3) If $f=v$, then for all $s>0$, the estimate (2.1) holds with $V(t)=\int_{0}^{t}\|\nabla v(\tau)\|_{L^{\infty}} \mathrm{d} \tau$.

(4) If $r<+\infty$, then $f \in C\left([0, T] ; B_{p, r}^{s}\right)$. If $r=+\infty$, then $f \in C\left([0, T] ; B_{p, r}^{s^{\prime}}\right)$ for all $s^{\prime}<s$.

Lemma 2.3 (Existence and uniqueness [20,21]). Let $\left(p, p_{1}, r\right) \in[1,+\infty]^{3}$ and $s>$ $-d \min \left\{\frac{1}{p_{1}}, \frac{1}{p^{\prime}}\right\}$ with $p^{\prime} \doteq\left(1-\frac{1}{p}\right)^{-1}$. Assume that $f_{0} \in B_{p, r}^{s}, F \in L^{1}\left([0, T] ; B_{p, r}^{s}\right)$. Let $v$ be a time-dependent vector field such that $v \in L^{\rho}\left([0, T] ; B_{\infty, \infty}^{-M}\right)$ for some $\rho>1, M>0$ and $\nabla v \in L^{1}\left([0, T] ; B_{p_{1}, r}^{\frac{d}{p_{1}}} \cap L^{\infty}\right)$ if $s<1+\frac{d}{p_{1}}$ and $\nabla v \in L^{1}\left([0, T] ; B_{p_{1}, r}^{s-1}\right)$ if $s>1+\frac{d}{1 p}$ or $s=1+\frac{d}{p_{1}}$ and $r=1$. Then the transport equations $(T)$ have a unique solution $f \in L^{\infty}\left([0, T] ; B_{p, r}^{s}\right) \cap\left(\cap_{s^{\prime}<s} C[0, T] ; B_{p, r}^{s^{\prime}}\right)$ and the inequalities in Lemma 2.2 hold. Moreover, $r<\infty$, then we have $\left.f \in C[0, T] ; B_{p, 1}^{s}\right)$.

Lemma 2.4 (A priori estimate in Sobolev spaces [26]) Let $0<\sigma<1$. Assume that $f_{0} \in$ $H^{\sigma}, F \in L^{1}\left(0, T ; H^{\sigma}\right)$, and $v, \partial_{x} v \in L^{1}\left(0, T ; L^{\infty}\right)$. If $f \in L^{\infty}\left(0, T ; H^{\sigma}\right) \cap C\left([0, T] ; \mathcal{S}^{\prime}\right)$ solves $(T)$, then $f \in C\left([0, T] ; H^{\sigma}\right)$ and there exists a constant $C$ depending only on $\sigma$ such that

$$
\|f\|_{H^{\sigma}} \leq\left\|f_{0}\right\|_{H^{\sigma}}+\int_{0}^{t}\|F(\tau)\|_{H^{\sigma}} \mathrm{d} \tau+C \int_{0}^{t} V^{\prime}(\tau)\|f(\tau)\|_{H^{\sigma}} \mathrm{d} \tau,
$$

with $V(t)=\int_{0}^{t}\left(\|v(\tau)\|_{L^{\infty}}+\left\|\partial_{x} v(\tau)\right\|_{\left.L^{\infty}\right) \mathrm{d} \tau}\right.$.

Lemma 2.5 (1D Moser-type estimates [20,21]) Assume that $1 \leq p, r \leq+\infty$, the following estimates hold:

(i) For $s>0$,

$$
\|f g\|_{B_{p, r}^{s}} \leq C\left(\|f\|_{B_{p, r}^{s}}\|g\|_{L^{\infty}}+\|g\|_{B_{p, r}^{s}}\|f\|_{L^{\infty}}\right) ;
$$

(ii) $\forall s_{1} \leq \frac{1}{p}<s_{2}\left(s_{2} \geq \frac{1}{p}\right.$ if $\left.r=1\right)$ and $s_{1}+s_{2}>0$, we have

$$
\|f g\|_{B_{p, r}^{s_{1}}} \leq C\|f\|_{B_{p, r}^{s_{1}}}\|g\|_{B_{p, r}^{s_{2}}}
$$

(iii) In Sobolev spaces $H^{s}=B_{2,2}^{s}$, we have for $s>0$,

$$
\left\|f \partial_{x} g\right\|_{H^{s}} \leq C\left(\|f\|_{H^{s+1}}\|g\|_{L^{\infty}}+\left\|\partial_{x} g\right\|_{H^{s}}\|f\|_{L^{\infty}}\right),
$$

where $C$ is a positive constant independent of $f$ and $g$.

Definition 2.2 For $T>0, s \in \mathbb{R}$ and $1 \leq p \leq+\infty$, we set

$$
\begin{aligned}
& E_{p, r}^{s}(T) \triangleq C\left([0, T] ; B_{p, r}^{s}\right) \cap C^{1}\left([0, T] ; B_{p, r}^{s-1}\right) \text { if } r<+\infty, \\
& E_{p, \infty}^{s}(T) \triangleq L^{\infty}\left([0, T] ; B_{p, \infty}^{s}\right) \cap \operatorname{Lip}\left([0, T] ; B_{p, \infty}^{s-1}\right) \\
& \text { and } E_{p, r}^{s} \triangleq \cap_{T>0} E_{p, r}^{s}(T) .
\end{aligned}
$$

We are now ready to state the first main result of the paper. 
Theorem 2.1 Let $p, r \in[1, \infty]$ and $s>\max \left\{\frac{5}{2}, 2+\frac{1}{p}\right\}$. Assume that $\left(u_{0}, v_{0}\right) \in B_{p, r}^{s} \times$ $B_{p, r}^{s}$. There exists a time $T>0$ such that the initial-value problem (1.1) has a unique solution $(u, v) \in E_{p, r}^{s}(T) \times E_{p, r}^{s}(T)$ and the map $\left(u_{0}, v_{0}\right) \mapsto(u, v)$ is continuous from $a$ neighborhood of $\left(u_{0}, v_{0}\right)$ in $B_{p, r}^{s} \times B_{p, r}^{s}$ into

$$
C\left([0, T] ; B_{p, r}^{s^{\prime}}\right) \cap C^{1}\left([0, T] ; B_{p, r}^{s^{\prime}-1}\right) \times C\left([0, T] ; B_{p, r}^{s^{\prime}}\right) \cap C^{1}\left([0, T] ; B_{p, r}^{s^{\prime}-1}\right)
$$

for every $s^{\prime}<s$ when $r=+\infty$ and $s^{\prime}=s$, whereas $r<+\infty$.

In the following, we denote $C>0$ a generic constant only depending on $p, r, s$. Uniqueness and continuity with respect to the initial data are an immediate consequence of the following result.

Proposition 2.3 Let $1 \leq p, r \leq+\infty$ and $s>\max \left\{\frac{5}{2}, 2+\frac{1}{p}\right\}$. Suppose that $\left(u^{(i)} ; v^{(i)}\right) \in$ $\left\{L^{\infty}\left([0, T] ; B_{p, r}^{s}\right) \cap C\left([0, T] ; \mathcal{S}^{\prime}\right)\right\}^{2}(i=1,2)$ be two given solutions of the initial-value problem (1.1) with the initial data $\left(u_{0}^{(i)} ; v_{0}^{(i)}\right) \in B_{p, r}^{s} \times B_{p, r}^{s}(i=1 ; 2)$. Then for every $t \in[0 ; T]$, we have

$$
\begin{aligned}
& \left\|u^{(1)}(t)-u^{(2)}(t)\right\|_{B_{p, r}^{s-1}}+\left\|v^{(1)}(t)-v^{(2)}(t)\right\|_{B_{p, r}^{s-1}} \\
& \quad \leq\left(\left\|u_{0}^{(1)}-u_{0}^{(2)}\right\|_{B_{p, r}^{s-1}}+\left\|v_{0}^{(1)}-v_{0}^{(2)}\right\|_{B_{p, r}^{s-1}}\right) \\
& \quad \times \exp \left\{C \int_{0}^{t}\left(\left\|u^{(1)}(\tau)\right\|_{B_{p, r}^{s}}^{2}+\left\|u^{(2)}(\tau)\right\|_{B_{p, r}^{s}}^{2}+\left\|v^{(1)}(\tau)\right\|_{B_{p, r}^{s}}^{2}+\left\|v^{(2)}(\tau)\right\|_{B_{p, r}^{s}}^{2}\right) \mathrm{d} \tau\right\} .
\end{aligned}
$$

Proof Denote $u^{(12)}=u^{(2)}-u^{(1)}, v^{(12)}=v^{(2)}-v^{(1)}, m^{(12)}=m^{(2)}-m^{(1)}$ and $n^{(12)}=$ $n^{(2)}-n^{(1)}$. It is obvious that

$$
u^{(12)}, v^{(12)} \in L^{\infty}\left([0, T] ; B_{p, r}^{s}\right) \cap C\left([0, T] ; \mathcal{S}^{\prime}\right),
$$

which implies that $u^{(12)}, v^{(12)} \in C\left([0, T] ; B_{p, r}^{s-1}\right)$ and $\left(u^{(12)}, v^{(12)}, m^{(12)}, n^{(12)}\right)$ solves the transport equations

$$
\left\{\begin{array}{l}
m_{t}^{(12)}+2 u^{(1)} v^{(1)} m_{x}^{(12)}=F \\
n_{t}^{(12)}+2 u^{(1)} v^{(1)} n_{x}^{(12)}=G
\end{array}\right.
$$

with

$$
\begin{aligned}
F= & -2\left(u^{(2)} v^{(12)}+u^{(12)} v^{(1)}\right) m_{x}^{(2)}-4\left(u_{x}^{(2)} v^{(12)} m^{(2)}+u_{x}^{(2)} v^{(1)} m^{(12)}+u_{x}^{(12)} v^{(1)} m^{(1)}\right) \\
& -2\left(v_{x}^{(2)} u^{(12)} m^{(2)}+v_{x}^{(2)} u^{(1)} m^{(12)}+v_{x}^{(12)} u^{(1)} m^{(1)}\right) \\
G= & -2\left(v^{(2)} u^{(12)}+v^{(12)} u^{(1)}\right) n_{x}^{(2)}-4\left(v_{x}^{(2)} u^{(12)} n^{(2)}+v_{x}^{(2)} u^{(1)} n^{(12)}+v_{x}^{(12)} u^{(1)} n^{(1)}\right) \\
& -2\left(u_{x}^{(2)} v^{(12)} n^{(2)}+u_{x}^{(2)} v^{(1)} n^{(12)}+u_{x}^{(12)} v^{(1)} n^{(1)}\right)
\end{aligned}
$$

According to Lemma 2.2, we have

$$
\begin{aligned}
& e^{-C \int_{0}^{t}\left\|\partial_{x}\left(u^{(1)} v^{(1)}\right)\left(\tau^{\prime}\right)\right\|_{B_{p, r}^{s-2}} \mathrm{~d} \tau^{\prime}}\left\|m^{(12)}(t)\right\|_{B_{p, r}^{s-3}} \\
& \quad \leq\left\|m_{0}^{(12)}\right\|_{B_{p, r}^{s-3}}+C \int_{0}^{t} e^{-C \int_{0}^{\tau}\left\|\partial_{x}\left(u^{(1)} v^{(1)}\right)\left(\tau^{\prime}\right)\right\|_{B_{p, r}^{s-2}} \mathrm{~d} \tau^{\prime}}\left(\|F\|_{B_{p, r}^{s-3}}\right) \mathrm{d} \tau,
\end{aligned}
$$

and

$$
e^{-C \int_{0}^{t}\left\|\partial_{x}\left(u^{(1)} v^{(1)}\right)\left(\tau^{\prime}\right)\right\|_{B_{p, r}^{s-2}} \mathrm{~d} \tau^{\prime}}\left\|n^{(12)}(t)\right\|_{B_{p, r}^{s-3}}
$$




$$
\leq\left\|n_{0}^{(12)}\right\|_{B_{p, r}^{s-3}}+C \int_{0}^{t} e^{-C \int_{0}^{\tau}\left\|\partial_{x}\left(u^{(1)} v^{(1)}\right)\left(\tau^{\prime}\right)\right\|_{B_{p, r}^{s-2}} \mathrm{~d} \tau^{\prime}}\left(\|G\|_{B_{p, r}^{s-3}}\right) \mathrm{d} \tau,
$$

For $s>\max \left\{\frac{5}{2}, 2+\frac{1}{p}\right\}$, by Lemma 2.4, we have

$$
\begin{aligned}
& \|F\|_{B_{p, r}^{s, 3}}=\|-2\left(u^{(2)} v^{(12)}+u^{(12)} v^{(1)}\right) m_{x}^{(2)}-4\left(u_{x}^{(2)} v^{(12)} m^{(2)}+u_{x}^{(2)} v^{(1)} m^{(12)}+u_{x}^{(12)} v^{(1)} m^{(1)}\right) \\
& -2\left(v_{x}^{(2)} u^{(12)} m^{(2)}+v_{x}^{(2)} u^{(1)} m^{(12)}+v_{x}^{(12)} u^{(1)} m^{(1)}\right) \|_{B_{p, r}^{s-3}} \\
& \leq C\left\|u^{(2)} v^{(12)}+u^{(12)} v^{(1)}\right\|_{B_{p, r}^{s-3}}\left\|m^{(2)}\right\|_{B_{p, r}^{s-2}}+C\left\|u^{(2)}\right\|_{B_{p, r}^{s-2}}\left\|v^{(12)} m^{(2)}\right\|_{B_{p, r}^{s-3}}
\end{aligned}
$$

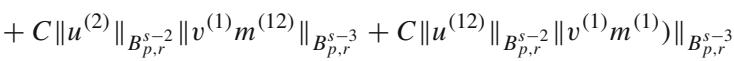

$$
\begin{aligned}
& +C\left\|v^{(2)}\right\|_{B_{p, r}^{s-2}}\left\|u^{(12)} m^{(2)}\right\|_{B_{p, r}^{s-3}}+C\left\|v^{(2)}\right\|_{B_{p, r}^{s-2}}\left\|u^{(1)} m^{(12)}\right\|_{B_{p, r}^{s-3}} \\
& \left.+C\left\|v^{(12)}\right\|_{B_{p, r}^{s-2}} \| u^{(1)} m^{(1)}\right) \|_{B_{p, r}^{s-3}} \\
& \leq C\left(\left\|u^{(12)}\right\|_{B_{p, r}^{s-1}}+\left\|v^{(12)}\right\|_{B_{p, r}^{s-1}}\right)\left(\left\|u^{(1)}\right\|_{B_{p, r}^{s}}^{2}+\left\|v^{(1)}\right\|_{B_{p, r}^{s}}^{2}+\left\|u^{(2)}\right\|_{B_{p, r}^{s}}^{2}+\left\|v^{(2)}\right\|_{B_{p, r}^{s}}^{2}\right), \\
& \|G\|_{B_{p, r}^{s, 3}}=\|-2\left(v^{(2)} u^{(12)}+v^{(12)} u^{(1)}\right) n_{x}^{(2)}-4\left(v_{x}^{(2)} u^{(12)} n^{(2)}+v_{x}^{(2)} u^{(1)} n^{(12)}+v_{x}^{(12)} u^{(1)} n^{(1)}\right) \\
& -2\left(u_{x}^{(2)} v^{(12)} n^{(2)}+u_{x}^{(2)} v^{(1)} n^{(12)}+u_{x}^{(12)} v^{(1)} n^{(1)}\right) \|_{B_{p, r}^{s-3}} \\
& \leq C\left\|v^{(2)} u^{(12)}+v^{(12)} u^{(1)}\right\|_{B_{p, r}^{s-3}}\left\|n^{(2)}\right\|_{B_{p, r}^{s-2}}+C\left\|v^{(2)}\right\|_{B_{p, r}^{s-2}}\left\|u^{(12)} m^{(2)}\right\|_{B_{p, r}^{s-3}}
\end{aligned}
$$

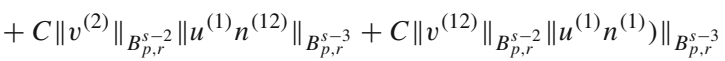

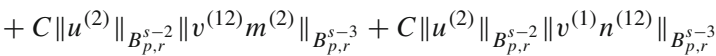

$$
\begin{aligned}
& \left.+C\left\|u^{(12)}\right\|_{B_{p, r}^{s-2}} \| v^{(1)} n^{(1)}\right) \|_{B_{p, r}^{s-3}} \\
& \leq C\left(\left\|v^{(12)}\right\|_{B_{p, r}^{s-1}}+\left\|u^{(12)}\right\|_{B_{p, r}^{s-1}}\right)\left(\left\|v^{(1)}\right\|_{B_{p, r}^{s}}^{2}+\left\|u^{(1)}\right\|_{B_{p, r}^{s}}^{2}+\left\|v^{(2)}\right\|_{B_{p, r}^{s}}^{2}+\left\|u^{(2)}\right\|_{B_{p, r}^{s}}^{2}\right) .
\end{aligned}
$$

Therefore, inserting the above estimates to (2.4)-(2.5), we obtain

$$
\begin{aligned}
& e^{-C \int_{0}^{t}\left\|\partial_{x}\left(u^{(1)} v^{(1)}\right)\left(\tau^{\prime}\right)\right\|_{B_{p, r}^{s-2}} d \tau^{\prime}}\left(\left\|u^{(12)}(t)\right\|_{B_{p, r}^{s-1}}+\left\|v^{(12)}(t)\right\|_{B_{p, r}^{s-1}}\right) \\
& \leq\left\|u_{0}^{(12)}\right\|_{B_{p, r}^{s-1}}+\left\|v_{0}^{(12)}\right\|_{B_{p, r}^{s-1}}+C \int_{0}^{t} e^{-C \int_{0}^{\tau}\left\|\partial_{x}\left(u^{(1)} v^{(1)}\right)\left(\tau^{\prime}\right)\right\|_{B_{p, r}^{s-2}} \mathrm{~d} \tau^{\prime}} \\
& \times\left(\left\|v^{(12)}\right\|_{B_{p, r}^{s-1}}+\left\|u^{(12)}\right\|_{B_{p, r}^{s-1}}\right)\left(\left\|v^{(1)}\right\|_{B_{p, r}^{s}}^{2}+\left\|u^{(1)}\right\|_{B_{p, r}^{s}}^{2}+\left\|v^{(2)}\right\|_{B_{p, r}^{s}}^{2}+\left\|u^{(2)}\right\|_{B_{p, r}^{s}}^{2}\right) \mathrm{d} \tau .
\end{aligned}
$$

Hence, thanks to

$$
\left\|\partial_{x}\left(u^{(1)} v^{(1)}\right)\right\|_{B_{p, r}^{s-2}} \leq C\left(\left\|u^{(1)}\right\|_{B_{p, r}^{s}}^{2}+\left\|v^{(1)}\right\|_{B_{p, r}^{s}}^{2}\right),
$$

and then applying the Gronwall's inequality, we reach (2.2).

Now let us start the proof of Theorem 1.1, which is motivated by the proof of local existence theorem about the Camassa-Holm equation in [20]. Firstly, we shall use the classical Friedrichs regularization method to construct the approximate solutions to the Cauchy problem (2.1).

Lemma 2.6 Assume that $u^{(0)}=v^{(0)}=0$. Let $1 \leq p, r \leq+\infty, s>\max \left\{\frac{5}{2}, 2+\frac{1}{p}\right\}$ and $u_{0}, v_{0} \in B_{p, r}^{s}$. Then there exists a sequence of smooth functions $\left(u^{(l)}, v^{(l)}\right)_{l \in \mathbb{N}} \in$ $C\left(R^{+} ; B_{p, r}^{\infty}\right)^{2}$ solving the following linear transport equation by induction

$$
\begin{cases}\left(\partial_{t}+2\left(u^{(l)} v^{(l)}\right) \partial_{x}\right) m^{(l+1)}=-4 v^{(l)} u_{x}^{(l)} m^{(l)}-2 u^{(l)} v_{x}^{(l)} m^{(l)}, & t>0, x \in \mathbb{R} \\ \left(\partial_{t}+2\left(u^{(l)} v^{(l)}\right) \partial_{x}\right) n^{(l+1)}=-4 u^{(l)} v_{x}^{(l)} n^{(l)}-2 v^{(l)} u_{x}^{(l)} n^{(l)}, & t>0, x \in \mathbb{R} \\ u^{(l+1)}(x, 0)=u_{0}^{(l+1)}(x)=S_{l+1} u_{0}, & x \in \mathbb{R}, \\ v^{(l+1)}(x, 0)=v_{0}^{(l+1)}(x)=S_{l+1} v_{0}, & x \in \mathbb{R} .\end{cases}
$$


Moreover, there is a positive T such that the solutions satisfied the following properties

(i) $\left(u^{(l)}, v^{(l)}\right)_{l \in \mathbb{N}}$ is uniformly bounded in $E_{p, r}^{s}(T) \times E_{p, r}^{s}(T)$.

(ii) $\left(u^{(l)}, v^{(l)}\right)_{l \in \mathbb{N}}$ is a Cauchy sequence in $C\left([0, T] ; B_{p, r}^{s-1}\right) \times C\left([0, T] ; B_{p, r}^{s-1}\right)$.

Proof Since all the data $S_{n+1} u_{0}$ and $S_{n+1} v_{0}$ belong to $B_{p, r}^{\infty}$, Lemma 2.3 enables us to show by induction that for all $l \in \mathbb{N}$, Eq. (2.6) has a global solution which belongs to $C\left(R^{+} ; B_{p, r}^{\infty}\right)^{2}$. Thanks to Lemma 2.2 and the proof of Proposition 2.3, we have the following inequality for all $l \in \mathbb{N}$

$$
\begin{aligned}
& e^{-C \int_{0}^{t}\left\|\left(u^{(l)} v^{(l)}\right)\left(\tau^{\prime}\right)\right\|_{B_{p, r}^{s-1}} \mathrm{~d} \tau^{\prime}}\left\|m^{(l+1)}(t)\right\|_{B_{p, r}^{s-2}} \\
& \quad \leq\left\|S_{l+1} u_{0}\right\|_{B_{p, r}^{s}}+C \int_{0}^{t} e^{-C \int_{0}^{\tau}\left\|\left(u^{(1)} v^{(1)}\right)\left(\tau^{\prime}\right)\right\|_{B_{p, r}^{s-1}} \mathrm{~d} \tau^{\prime}}\left\|-4 v^{(l)} u_{x}^{(l)} m^{(l)}-2 u^{(l)} v_{x}^{(l)} m^{(l)}\right\|_{B_{p, r}^{s-2}} \mathrm{~d} \tau,
\end{aligned}
$$

and

$$
\begin{aligned}
& e^{-C \int_{0}^{t}\left\|\left(u^{(l)} v^{(l)}\right)\left(\tau^{\prime}\right)\right\|_{B_{p, r}^{s-1}} \mathrm{~d} \tau^{\prime}}\left\|n^{(l+1)}(t)\right\|_{B_{p, r}^{s-2}} \\
& \quad \leq\left\|S_{l+1} v_{0}\right\|_{B_{p, r}^{s}}+C \int_{0}^{t} e^{-C \int_{0}^{\tau}\left\|\left(u^{(1)} v^{(1)}\right)\left(\tau^{\prime}\right)\right\|_{B_{p, r}^{s-1}} \mathrm{~d} \tau^{\prime}}\left\|-4 u^{(l)} v_{x}^{(l)} n^{(l)}-2 v^{(l)} u_{x}^{(l)} n^{(l)}\right\|_{B_{p, r}^{s-2}} \mathrm{~d} \tau,
\end{aligned}
$$

Thanks to $s>\max \left\{\frac{5}{2}, 2+\frac{1}{p}\right\}$, we find $B_{p, r}^{s-2}$ is an algebra. From this, one obtains

$$
\begin{aligned}
& \left\|v^{(l)} u_{x}^{(l)} m^{(l)}\right\|_{B_{p, r}^{s-2}} \leq C\left\|v^{(l)}\right\|_{B_{p, r}^{s-2}}\left\|m^{(l)}\right\|_{B_{p, r}^{s-2}}\left\|u_{x}^{(l)}\right\|_{B_{p, r}^{s-2}} \leq C\left(\left\|u^{(l)}\right\|_{B_{p, r}^{s}}+\left\|v^{(l)}\right\|_{B_{p, r}^{s}}\right)^{3}, \\
& \left\|u^{(l)} v_{x}^{(l)} n^{(l)}\right\|_{B_{p, r}^{s-2}} \leq C\left\|u^{(l)}\right\|_{B_{p, r}^{s-2}}\left\|n^{(l)}\right\|_{B_{p, r}^{s-2}}\left\|v_{x}^{(l)}\right\|_{B_{p, r}^{s-2}} \leq\left(\left\|v^{(l)}\right\|_{B_{p, r}^{s}}+\left\|u^{(l)}\right\|_{B_{p, r}^{s}}\right)^{3}, \\
& \left\|u^{(l)} v_{x}^{(l)} m^{(l)}\right\|_{B_{p, r}^{s-2}} \leq C\left\|u^{(l)}\right\|_{B_{p, r}^{s-2}}\left\|m^{(l)}\right\|_{B_{p, r}^{s-2}}\left\|v_{x}^{(l)}\right\|_{B_{p, r}^{s-2}} \leq C\left(\left\|v^{(l)}\right\|_{B_{p, r}^{s}}+\left\|u^{(l)}\right\|_{B_{p, r}^{s}}\right)^{3}, \\
& \left\|v^{(l)} u_{x}^{(l)} n^{(l)}\right\|_{B_{p, r}^{s-2}} \leq C\left\|v^{(l)}\right\|_{B_{p, r}^{s-2}}\left\|n^{(l)}\right\|_{B_{p, r}^{s-2}}\left\|u_{x}^{(l)}\right\|_{B_{p, r}^{s-2}} \leq\left(\left\|u^{(l)}\right\|_{B_{p, r}^{s}}+\left\|v^{(l)}\right\|_{B_{p, r}^{s}}\right)^{3},
\end{aligned}
$$

which along with the above inequality leads to

$$
\begin{aligned}
& e^{-C \int_{0}^{t}\left\|\left(u^{(l)} v^{(l)}\right)\left(\tau^{\prime}\right)\right\|_{B_{p, r}^{s-1}} \mathrm{~d} \tau^{\prime}}\left(\left\|u^{(l+1)}(t)\right\|_{B_{p, r}^{s}}+\left\|v^{(l+1)}(t)\right\|_{B_{p, r}^{s}}\right) \\
& \quad \leq\left\|u_{0}\right\|_{B_{p, r}^{s}}+\left\|v_{0}\right\|_{B_{p, r}^{s}}+C \int_{0}^{t} e^{-C \int_{0}^{\tau}\left\|\left(u^{(1)} v^{(1)}\right)\left(\tau^{\prime}\right)\right\|_{B_{p, r}^{s-1}} \mathrm{~d} \tau^{\prime}}\left(\left\|u^{(l)}\right\|_{B_{p, r}^{s}}+\left\|v^{(l)}\right\|_{B_{p, r}^{s}}\right)^{3} \mathrm{~d} \tau .
\end{aligned}
$$

Let us choose a $T>0$ such that $4 C\left(\left\|u_{0}\right\|_{B_{p, r}^{s}}+\left\|v_{0}\right\|_{B_{p, r}^{s}}\right)^{2} T<1$, and suppose by induction that for all $t \in[0, T]$

$$
\left\|u^{(l)}(t)\right\|_{B_{p, r}^{s}}+\left\|v^{(l)}(t)\right\|_{B_{p, r}^{s}} \leq \frac{\left\|u_{0}\right\|_{B_{p, r}^{s}}+\left\|v_{0}\right\|_{B_{p, r}^{s}}}{\left(1-4 C\left(\left\|u_{0}\right\|_{B_{p, r}^{s}}+\left\|v_{0}\right\|_{B_{p, r}^{s}}\right)^{2} t\right)^{\frac{1}{2}}} .
$$

Indeed, since $B_{p, r}^{s-1}$ is an algebra, one obtains from (2.8) that for any $0<\tau<t$

$$
C \int_{\tau}^{t}\left\|\partial_{x}\left(u^{(l)} v^{(l)}\right)\left(\tau^{\prime}\right)\right\|_{B_{p, r}^{s-1}} \mathrm{~d} \tau^{\prime} \leq C \int_{\tau}^{t}\left(\left\|u^{(l)}(t)\right\|_{B_{p, r}^{s}}+\left\|v^{(l)}(t)\right\|_{B_{p, r}^{s}}\right)^{2} \mathrm{~d} \tau
$$




$$
\begin{aligned}
& \leq C \int_{\tau}^{t} \frac{\left(\left\|u_{0}\right\|_{B_{p, r}^{s}}+\left\|v_{0}\right\|_{B_{p, r}^{s}}\right)^{2}}{1-4 C\left(\left\|u_{0}\right\|_{B_{p, r}^{s}}+\left\|v_{0}\right\|_{B_{p, r}^{s}}\right)^{2} t} \mathrm{~d} \tau \\
& =\frac{1}{4} \ln \left(1-4 C\left(\left\|u_{0}\right\|_{B_{p, r}^{s}}+\left\|v_{0}\right\|_{B_{p, r}^{s}}\right)^{2} \tau\right) \\
& -\frac{1}{4} \ln \left(1-4 C\left(\left\|u_{0}\right\|_{B_{p, r}^{s}}+\left\|v_{0}\right\|_{B_{p, r}^{s}}\right)^{2} t\right) .
\end{aligned}
$$

And then inserting (2.9) and (2.8) into (2.7) leads to

$$
\begin{aligned}
& \left\|u^{(l+1)}(t)\right\|_{B_{p, r}^{s}}+\left\|v^{(l+1)}(t)\right\|_{B_{p, r}^{s}} \\
& \leq \frac{\left\|u_{0}\right\|_{B_{p, r}^{s}}+\left\|v_{0}\right\|_{B_{p, r}^{s}}}{\left(1-4 C\left(\left\|u_{0}\right\|_{B_{p, r}^{s}}+\left\|v_{0}\right\|_{B_{p, r}^{s}}\right)^{2} t\right)^{\frac{1}{4}}}+\frac{C}{\left(1-4 C\left(\left\|u_{0}\right\|_{B_{p, r}^{s}}+\left\|v_{0}\right\|_{B_{p, r}^{s}}\right)^{2} t\right)^{\frac{1}{4}}} \\
& \quad \times \int_{0}^{t}\left(1-4 C\left(\left\|u_{0}\right\|_{B_{p, r}^{s}}+\left\|v_{0}\right\|_{B_{p, r}^{s}}\right)^{2} \tau\right)^{\frac{1}{4}} \frac{\left(\left\|u_{0}\right\|_{B_{p, r}^{s}}+\left\|v_{0}\right\|_{B_{p, r}^{s}}\right)^{2}}{\left(1-4 C\left(\left\|u_{0}\right\|_{B_{p, r}^{s}}+\left\|v_{0}\right\|_{B_{p, r}^{s}}\right)^{2} \tau\right)^{\frac{3}{2}}} \mathrm{~d} \tau \\
& \leq \frac{\left\|u_{0}\right\|_{B_{p, r}^{s}}+\left\|v_{0}\right\|_{B_{p, r}^{s}}}{\left(1-4 C\left(\left\|u_{0}\right\|_{B_{p, r}^{s}}+\left\|v_{0}\right\|_{B_{p, r}^{s}}\right)^{2} t\right)^{\frac{1}{4}}}\left(1+C \int_{0}^{t} \frac{\left(\left\|u_{0}\right\|_{B_{p, r}^{s}}+\left\|v_{0}\right\|_{B_{p, r}^{s}}\right)^{2}}{\left(1-4 C\left(\left\|u_{0}\right\|_{B_{p, r}^{s}}+\left\|v_{0}\right\|_{B_{p, r}^{s}}\right)^{2} t\right)^{\frac{5}{4}}} \mathrm{~d} \tau\right) \\
& =\frac{\left\|u_{0}\right\|_{B_{p, r}^{s}}+\left\|v_{0}\right\|_{B_{p, r}^{s}}}{\left(1-4 C\left(\left\|u_{0}\right\|_{B_{p, r}^{s}}+\left\|v_{0}\right\|_{B_{p, r}^{s}}\right)^{2} t\right)^{\frac{1}{2}}} .
\end{aligned}
$$

Hence, one can see that

$$
\left\|u^{(l+1)}(t)\right\|_{B_{p, r}^{s}}+\left\|v^{(l+1)}(t)\right\|_{B_{p, r}^{s}} \leq \frac{\left\|u_{0}\right\|_{B_{p, r}^{s}}+\left\|v_{0}\right\|_{B_{p, r}^{s}}}{\left(1-4 C\left(\left\|u_{0}\right\|_{B_{p, r}^{s}}+\left\|v_{0}\right\|_{B_{p, r}^{s}}\right)^{2} t\right)^{\frac{1}{2}}},
$$

which implies that $\left(u^{(l)}, v^{(l)}\right)_{l \in \mathbb{N}}$ is uniformly bounded in $C\left([0 ; T] ; B_{p, r}^{s}\right) \times C\left([0 ; T] ; B_{p, r}^{s}\right)$. Using the Moser-type estimates (see Lemma 2.4), one finds that

$$
\begin{aligned}
& \left\|u^{(l)} v^{(l)} \partial_{x} m^{(l+1)}\right\|_{B_{p, r}^{s-3}} \leq C\left\|u^{(l+1)}\right\|_{B_{p, r}^{s}}\left(\left\|u^{(l)}\right\|_{B_{p, r}^{s}}^{2}+\left\|v^{(l)}\right\|_{B_{p, r}^{s}}^{2}\right), \\
& \left\|v^{(l)} u^{(l)} \partial_{x} n^{(l+1)}\right\|_{B_{p, r}^{s-3}} \leq C\left\|v^{(l+1)}\right\|_{B_{p, r}^{s}}\left(\left\|u^{(l)}\right\|_{B_{p, r}^{s}}^{2}+\left\|v^{(l)}\right\|_{B_{p, r}^{s}}^{2}\right),
\end{aligned}
$$

and

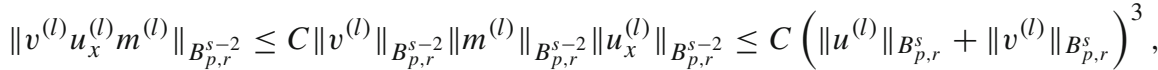

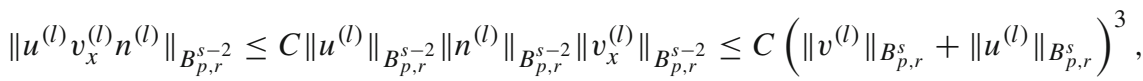

$$
\begin{aligned}
& \left\|u^{(l)} v_{x}^{(l)} m^{(l)}\right\|_{B_{p, r}^{s-2}} \leq C\left\|u^{(l)}\right\|_{B_{p, r}^{s-2} \|}\left\|m^{(l)}\right\|_{B_{p, r}^{s-2}}\left\|v_{x}^{(l)}\right\|_{B_{p, r}^{s-2}} \leq C\left(\left\|v^{(l)}\right\|_{B_{p, r}^{s}}+\left\|u^{(l)}\right\|_{B_{p, r}^{s}}\right)^{3},
\end{aligned}
$$

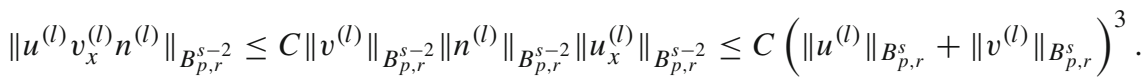


Hence, using Eq. (2.6), we have

$$
\left(\partial_{x} u^{(l+1)}, \partial_{x} v^{(l+1)}\right)_{l \in \mathbb{N}} \in C\left([0 ; T] ; B_{p, r}^{s-1}\right) \times C\left([0 ; T] ; B_{p, r}^{s-1}\right)
$$

uniformly bounded, which yields that the sequence $\left(u^{(l)}, v^{(l)}\right)_{\in \mathbb{N}}$ is uniformly bounded in $E_{p, r}^{s}(T) \times E_{p, r}^{s}(T)$.

Now, it suffices to show that $\left(u^{(l)}, v^{(l)}\right)_{l \in \mathbb{N}}$ is a Cauchy sequence in $C\left([0 ; T] ; B_{p, r}^{s-1}\right) \times$ $C\left([0 ; T] ; B_{p, r}^{s-1}\right)$. In fact, for all $l, k \in \mathbb{N}$, from (2.6), we have

$$
\left\{\begin{array}{l}
\left(\partial_{t}+2\left(u^{(l+k)} v^{(l+k)}\right) \partial_{x}\right)\left(m^{(l+k+1)}-m^{(l+1)}\right)=F^{\prime}, \\
\left(\partial_{t}+2\left(u^{(l+k)} v^{(l+k)}\right) \partial_{x}\right)\left(n^{(l+k+1)}-n^{(l+1)}\right)=G^{\prime},
\end{array}\right.
$$

with

$$
\begin{aligned}
F^{\prime}= & -2\left(u^{(l+k)}\left(v^{(l+k)}-v^{(l)}\right)+\left(u^{(l+k)}-u^{(l)}\right) v^{(l)}\right) m_{x}^{(l+1)}-4\left(u_{x}^{(k+l)}\left(v^{(k+l)}-v^{(l)}\right) m^{(k+l)}\right. \\
& \left.+u_{x}^{(k+l)} v^{(l)}\left(m^{(k+l)}-m^{(l)}\right)+\left(u_{x}^{(k+l)}-u_{x}^{(l)}\right) v^{(l)} m^{(l)}\right)-2\left(v_{x}^{(k+l)}\left(u^{(k+l)}-u^{(l)}\right) m^{(k+l)}\right. \\
& \left.+v_{x}^{(k+l)} u^{(l)}\left(m^{(k+l)}-m^{(l)}\right)+\left(v_{x}^{(k+l)}-v_{x}^{(l)}\right) u^{(l)} m^{(l)}\right), \\
G^{\prime}= & -2\left(v^{(l+k)}\left(u^{(l+k)}-u^{(l)}\right)+\left(v^{(l+k)}-v^{(l)}\right) u^{(l)}\right) n_{x}^{(l+1)}-3\left(v_{x}^{(k+l)}\left(u^{(k+l)}-u^{(l)}\right) n^{(k+l)}\right. \\
& \left.+v_{x}^{(k+l)} u^{(l)}\left(n^{(k+l)}-n^{(l)}\right)+\left(v_{x}^{(k+l)}-v_{x}^{(l)}\right) u^{(l)} n^{(l)}\right)-3\left(u_{x}^{(k+l)}\left(v^{(k+l)}-u^{(l)}\right) n^{(k+l)}\right. \\
& \left.+u_{x}^{(k+l)} v^{(l)}\left(n^{(k+l)}-n^{(l)}\right)+\left(u_{x}^{(k+l)}-u_{x}^{(l)}\right) v^{(l)} n^{(l)}\right) .
\end{aligned}
$$

Similar to the proof of Proposition 2.3, then for every $t \in[0, T]$, we obtain

$$
\begin{aligned}
& e^{-C \int_{0}^{t}\left\|\partial_{x}\left(u^{(k+l)} v^{(k+l)}\right)\left(\tau^{\prime}\right)\right\|_{B_{p, r}^{s-2}} \mathrm{~d} \tau^{\prime}}\left(\left\|\left(u^{(k+l+1)}-u^{(l+1)}\right)(t)\right\|_{B_{p, r}^{s-1}}+\left\|\left(v^{(k+l+1)}-v^{l+1}\right)(t)\right\|_{B_{p, r}^{s-1}}\right) \\
& \left.\left.\leq \| u_{0}^{(k+l+1)}-u_{0}^{(l+1)}\right)\left\|_{B_{p, r}^{s-1}}+\right\| u_{0}^{(k+l+1)}-u_{0}^{(l+1)}\right) \|_{B_{p, r}^{s-1}}+C \int_{0}^{t} e^{-C \int_{0}^{\tau}\left\|\partial_{x}\left(u^{(l)} v^{(l)}\right)\left(\tau^{\prime}\right)\right\|_{B_{p, r}^{s-2}} \mathrm{~d} \tau^{\prime}} \\
& \quad \times\left(\left\|v^{(k+l)}-v^{(l)}\right\|_{B_{p, r}^{s-1}}+\left\|v^{(k+l)}-v^{(l)}\right\|_{B_{p, r}^{s-1}}\right)\left(\left\|v^{(l)}\right\|_{B_{p, r}^{s}}^{2}+\left\|u^{(l)}\right\|_{B_{p, r}^{s}}^{2}+\left\|v^{(l+k)}\right\|_{B_{p, r}^{s}}^{2}\right. \\
& \left.\quad+\left\|u^{(k+l)}\right\|_{B_{p, r}^{s}}^{2}+\left\|u^{(l+1)}\right\|_{B_{p, r}^{s}}^{2}+\left\|v^{(l+1)}\right\|_{B_{p, r}^{s}}^{2}\right) \mathrm{d} \tau .
\end{aligned}
$$

Since $\left(u^{(l)}, v^{(l)}\right)_{l \in \mathbb{N}}$ is uniformly bounded in $E_{p, r}^{s}(T) \times E_{p, r}^{s}(T)$ and

$$
\begin{aligned}
& u_{0}^{(l+k+1)}-u_{0}^{(l+1)}=S_{k+l+1} u_{0}-S_{l+1} u_{0}=\sum_{q=l+1}^{l+k} \Delta_{q} u_{0}, \\
& v_{0}^{(l+k+1)}-v_{0}^{(l+1)}=S_{k+l+1} v_{0}-S_{l+1} v_{0}=\sum_{q=l+1}^{l+k} \Delta_{q} v_{0},
\end{aligned}
$$

we get a constant $C_{T}$ independent of $l, k$ such that for all $t \in[0, T]$

$$
\begin{aligned}
& \left\|\left(u^{(k+l+1)}-u^{(l+1)}\right)(t)\right\|_{B_{p, r}^{s-1}}+\left\|\left(v^{(k+l+1)}-v^{(l+1)}\right)(t)\right\|_{B_{p, r}^{s-1}} \\
& \quad \leq C_{T}\left(2^{-n}+\int_{0}^{t}\left(\left\|\left(u^{(k+l)}-u^{(l)}\right)(\tau)\right\|_{B_{p, r}^{s-1}}+\left\|\left(v^{(k+l)}-u^{(l)}\right)(\tau)\right\|_{B_{p, r}^{s-1}}\right) \mathrm{d} \tau\right) .
\end{aligned}
$$

Arguing by induction with respect to the index $l$, one can easily prove that

$$
\left\|\left(u^{(k+l+1)}-u^{(l+1)}\right)(t)\right\|_{L_{T}^{\infty}\left(B_{p, r}^{s-1}\right)}+\left\|\left(v^{(k+l+1)}-v^{(l+1)}\right)(t)\right\|_{L_{T}^{\infty}\left(B_{p, r}^{s-1}\right)}
$$




$$
\leq \frac{\left(T C_{T}\right)^{l+1}}{(l+1) !}\left(\left\|u^{k}\right\|_{L_{T}^{\infty}\left(B_{p, r}^{s-1}\right)}+\left\|v^{k}\right\|_{L_{T}^{\infty}\left(B_{p, r}^{s-1}\right)}\right)+C_{T} \sum_{q=0}^{l} 2^{q-l} \frac{\left(T C_{T}\right)^{q}}{q !}
$$

As $\left\|u^{(k)}\right\|_{L_{T}^{\infty}\left(B_{p, r}^{s-1}\right)},\left\|v^{(k)}\right\|_{L_{T}^{\infty}\left(B_{p, r}^{s-1}\right)}$ and $C$ are bounded independently of $k$, there exists constant $C_{T}^{\prime}$ independent of $l, k$ such that

$$
\left\|\left(u^{(k+l+1)}-u^{(l+1)}\right)(t)\right\|_{L_{T}^{\infty}\left(B_{p, r}^{s-1}\right)}+\left\|\left(v^{(k+l+1)}-v^{(l+1)}\right)(t)\right\|_{L_{T}^{\infty}\left(B_{p, r}^{s-1}\right)} \leq C_{T}^{\prime} 2^{-n} .
$$

Thus, $\left(u^{(l)}, v^{(l)}\right)_{n \in \mathbb{N}}$ is a Cauchy sequence in $C\left([0, T] ; B_{p, r}^{s-1}\right) \times\left([0, T] ; B_{p, r}^{s-1}\right)$.

Proof of Theorem 2.1. Thanks to Lemma 2.6, we obtain that $\left(u^{(l)}, v^{(l)}\right)_{l \in \mathbb{N}}$ is a Cauchy sequence in $C\left([0, T] ; B_{p, r}^{s-1}\right) \times C\left([0, T] ; B_{p, r}^{s-1}\right)$, so it converges to some function $(u, v) \in$ $C\left([0, T] ; B_{p, r}^{s-1}\right) \times C\left([0, T] ; B_{p, r}^{s-1}\right)$. We now have to check that $(u, v)$ belongs to $E_{p, r}^{s}(T) \times$ $E_{p, r}^{s}(T)$ and solves the Cauchy problem (1.1). Since $\left(u^{(l)}, v^{(l)}\right)_{l \in \mathbb{N}}$ is uniformly bounded in $L^{\infty}\left([0, T] ; C\left([0, T] ; B_{p, r}^{s}\right) \times L^{\infty}\left([0, T] ; C\left([0, T] ; B_{p, r}^{s}\right)\right.\right.$ according to Lemma 2.5 , the Fatou property for the Besov spaces (Proposition 2.2) guarantees that $(u, v)$ also belongs to

$$
L^{\infty}\left([0, T] ; C\left([0, T] ; B_{p, r}^{s}\right) \times L^{\infty}\left([0, T] ; C\left([0, T] ; B_{p, r}^{s}\right) .\right.\right.
$$

On the other hand, as $\left(u^{(l)}, v^{(l)}\right)_{l \in \mathbb{N}}$ converges to $(u, v)$ in $C\left([0, T] ; B_{p, r}^{s-1}\right) \times C\left([0, T] ; B_{p, r}^{s-1}\right)$, an interpolation argument ensures that the convergence holds in $C\left([0, T] ; B_{p, r}^{s^{\prime}}\right) \times$ $C\left([0, T] ; B_{p, r}^{s^{\prime}}\right)$, for any $s^{\prime}<s$. It is then easy to pass to the limit in Eq. (2.6) and to conclude that $(u, v)$ is indeed a solution to the Cauchy problem (1.1). Thanks to the fact that $u$ belongs to $L^{\infty}\left([0, T] ; C\left([0, T] ; B_{p, r}^{s}\right) \times L^{\infty}\left([0, T] ; C\left([0, T] ; B_{p, r}^{s}\right)\right.\right.$, the right-hand side of the equation

$$
\partial_{t} m+2 u v \partial_{x} m=-4 v u_{x} m-2 u v_{x} m
$$

belongs to $L^{\infty}\left([0, T] ; C\left([0, T] ; B_{p, r}^{s}\right)\right.$, and the right-hand side of the equation

$$
\partial_{t} m+2 u v \partial_{x} n=-4 u v_{x} n-2 v u_{x} n
$$

belongs to $L^{\infty}\left([0, T] ; C\left([0, T] ; B_{p, r}^{s}\right)\right.$ In particular, for the case $r<\infty$, Lemma 2.3 enables us to conclude that $(u, v) \in C\left([0, T] ; B_{p, r}^{s^{\prime}}\right) \times C\left([0, T] ; B_{p, r}^{s^{\prime}}\right)$ for any $s^{\prime}<s$. Finally, using the equation again, we see that $\left(\partial_{t} u, \partial_{t} v\right) \in C\left([0, T] ; B_{p, r}^{s^{\prime}}\right) \times C\left([0, T] ; B_{p, r}^{s^{\prime}}\right)$ if $r<\infty$, and in $L^{\infty}\left([0, T] ; C\left([0, T] ; B_{p, r}^{s-1}\right) \times L^{\infty}\left([0, T] ; C\left([0, T] ; B_{p, r}^{s-1}\right)\right.\right.$ otherwise. Therefore, $(u, v)$ belongs to $E_{p, r}^{s}(T) \times E_{p, r}^{s}(T)$. Moreover, a standard use of a sequence of viscosity approximate solutions $\left(u_{\varepsilon}, v_{\varepsilon}\right)_{\varepsilon>0}$ for the Cauchy problem (1.1) which converges uniformly in $C\left([0, T] ; B_{p, r}^{s}\right) \cap C^{1}\left([0, T] ; B_{p, r}^{s-1}\right) \times C\left([0, T] ; B_{p, r}^{s}\right) \cap C^{1}\left([0, T] ; B_{p, r}^{s-1}\right)$ gives the continuity of the solution $(u, v)$ in $E_{p, r}^{s} \times E_{p, r}^{s}$. The proof of Theorem 1.1 is complete.

\section{Blow-up criteria}

In this section, we derive the precise blow-up scenario of strong solutions to the system (1.1).

We are now ready to state the second main result of the paper.

Theorem 3.1 Let $\left(m_{0}, n_{0}\right) \in H^{s}(\mathbb{R}) \times H^{s}(\mathbb{R})$ with $s>\frac{1}{2}$ and $T$ be the maximal existence time of the solution $(m, n)$ to the system $(1.1)$. If $T<\infty$, then

$$
\int_{x}^{T}\left(\|m(t)\|_{L^{\infty}}+\|n(t)\|_{L^{\infty}}\right)^{2} \mathrm{~d} t=\infty .
$$


Proof We will prove the theorem by induction with respect to the regular index $s\left(s>\frac{1}{2}\right)$ as follows.

Step 1. For $s \in\left(\frac{1}{2}, 1\right)$, by Lemma 2.4 and the system (1.1), we have

$$
\begin{aligned}
\|m\|_{H^{s}} \leq & \left\|m_{0}\right\|_{H^{s}}+C \int_{0}^{t}\left(\|u v\|_{L^{\infty}}+\left\|u_{x} v+v_{x} u\right\|_{L^{\infty}}\right)\|m(\tau)\|_{H^{s}} \mathrm{~d} \tau \\
& +C \int_{0}^{t}\left\|-4 v u_{x} m-2 u v_{x} m\right\|_{H^{s}} \mathrm{~d} \tau \\
\|n\|_{H^{s}} \leq & \left\|n_{0}\right\|_{H^{s}}+C \int_{0}^{t}\left(\|u v\|_{L^{\infty}}+\left\|u_{x} v+v_{x} u\right\|_{L^{\infty}}\right)\|n(\tau)\|_{H^{s}} \mathrm{~d} \tau \\
& +C \int_{0}^{t}\left\|-4 u v_{x} n-2 v u_{x} n\right\|_{H^{s}} \mathrm{~d} \tau,
\end{aligned}
$$

for all $0<t<T$.

Noting that $u=\left(1-\partial_{x}^{2}\right)^{-1} m=p * m$ with $p(x)=\frac{1}{2} e^{-|x|}(x \in \mathbb{R}), u_{x}=\partial_{x} p * m, u_{x x}=$ $u-m$ and $\|p\|_{L^{1}}=\left\|\partial_{x} p\right\|_{L^{1}}=1$, together with the Young inequality implies that for all $s \in \mathbb{R}$,

$$
\begin{aligned}
& \|u\|_{L^{\infty}},\left\|u_{x}\right\|_{L^{\infty}},\left\|u_{x x}\right\|_{L^{\infty}} \leq C\|m\|_{L^{\infty}}, \\
& \|u\|_{H^{s}},\left\|u_{x}\right\|_{H^{s}},\left\|u_{x x}\right\|_{H^{s}} \leq C\|m\|_{H^{s}} .
\end{aligned}
$$

Similarly, we obtain

$$
\begin{aligned}
& \|v\|_{L^{\infty}},\left\|v_{x}\right\|_{L^{\infty}},\left\|v_{x x}\right\|_{L^{\infty}} \leq C\|n\|_{L^{\infty}}, \\
& \|v\|_{H^{s}},\left\|v_{x}\right\|_{H^{s}},\left\|v_{x x}\right\|_{H^{s}} \leq C\|n\|_{H^{s}} .
\end{aligned}
$$

Owing to the first estimate in Lemma 2.5 and (3.2)-(3.3), one has

$$
\begin{aligned}
\left\|-4 v u_{x} m-2 u v_{x} m\right\|_{H^{s}} & \leq C\left(\left\|4 v u_{x}+2 u v_{x}\right\|_{L^{\infty}}\|m\|_{H^{s}}+\left\|4 v u_{x}+2 u v_{x}\right\|_{H^{s}}\|m\|_{L^{\infty}}\right), \\
& \leq C\left(\|m\|_{L^{\infty}}\|n\|_{L^{\infty}}\|m\|_{H^{s}}+\|m\|_{L^{\infty}}^{2}\|n\|_{H^{s}}\right)
\end{aligned}
$$

and

$$
\|u v\|_{L^{\infty}}+\left\|u_{x} v+v_{x} u\right\|_{L^{\infty}} \leq C\|m\|_{L^{\infty}}\|n\|_{L^{\infty}} .
$$

Thus, we have

$$
\|m\|_{H^{s}} \leq\left\|m_{0}\right\|_{H^{s}}+C \int_{0}^{t}\left(\|m\|_{L^{\infty}}\|n\|_{L^{\infty}}\|m\|_{H^{s}}+\|m\|_{L^{\infty}}^{2}\|n\|_{H^{s}}\right) \mathrm{d} \tau .
$$

Similarly, we have

$$
\|n\|_{H^{s}} \leq\left\|n_{0}\right\|_{H^{s}}+C \int_{0}^{t}\left(\|n\|_{L^{\infty}}\|m\|_{L^{\infty}}\|n\|_{H^{s}}+\|n\|_{L^{\infty}}^{2}\|m\|_{H^{s}}\right) \mathrm{d} \tau .
$$

Thus, we have

$$
\begin{aligned}
& \|m\|_{H^{s}}+\|n\|_{H^{s}} \\
& \leq\left\|m_{0}\right\|_{H^{s}}+\mid n_{0} \|_{H^{s}}+C \int_{0}^{t}\left(\|m(\tau)\|_{L^{\infty}}+\|n(\tau)\|_{L^{\infty}}\right)^{2}\left(\|m(\tau)\|_{H^{s}}+\|n(\tau)\|_{H^{s}} \|_{H^{s}}\right) \mathrm{d} \tau .
\end{aligned}
$$


Taking advantage of Gronwall's inequality, one gets

$$
\|m\|\left\|_{H^{s}}+\right\| n \|_{H^{s}} \leq\left(\left\|m_{0}\right\|_{H^{s}}+\mid n_{0} \|_{H^{s}}\right) e^{C \int_{0}^{t}\left(\|m(\tau)\|_{L^{\infty}}+\|n(\tau)\|_{L^{\infty}}\right)^{2} \mathrm{~d} \tau} .
$$

Therefore, if the maximal existence time $T<\infty$ satisfies

$$
\int_{0}^{T}\left(\|m(\tau)\|_{L^{\infty}}+\|n(\tau)\|_{L^{\infty}}\right)^{2} \mathrm{~d} \tau<\infty
$$

the inequality (3.9) implies that

$$
\limsup _{t \rightarrow T}\left(\|m(t)\|_{H^{s}}+\|n(t)\|_{H^{s}}\right)<\infty,
$$

which contradicts the assumption that $T<\infty$ is the maximal existence time. This completes the proof of the theorem for $s \in\left(\frac{1}{2}, 1\right)$.

Step 2. For $s \in\left[1, \frac{3}{2}\right.$ ), applying Lemma 2.1 to the first equation of the system (1.1), we have

$$
\begin{aligned}
\|m\|_{H^{s}} \leq & \left\|m_{0}\right\|_{H^{s}}+C \int_{0}^{t}\left\|u_{x} v+v_{x} u\right\|_{H^{\frac{1}{2}} \cap L^{\infty}}\|m(\tau)\|_{H^{s}} \mathrm{~d} \tau \\
& +C \int_{0}^{t}\left\|-4 v u_{x} m-2 u v_{x} m\right\|_{H^{s}} \mathrm{~d} \tau .
\end{aligned}
$$

Noticing that

$$
\left\|u_{x} v+v_{x} u\right\|_{H^{\frac{1}{2}} \cap L^{\infty}} \leq C\left\|u_{x} v+v_{x} u\right\|_{H^{\frac{1}{2}+\varepsilon_{0}}} \leq C\|m\|_{H^{\frac{1}{2}+\varepsilon_{0}}}\|n\|_{H^{\frac{1}{2}+\varepsilon_{0}}},
$$

where $\varepsilon_{0} \in\left(0, \frac{1}{2}\right)$. Using (3.4) and the fact that $H^{\frac{1}{2}+\varepsilon_{0}}(\mathbb{R}) \hookrightarrow H^{\frac{1}{2}}(\mathbb{R}) \cap L^{\infty}(\mathbb{R})$ leads to

$$
\|m\|_{H^{s}} \leq\left\|m_{0}\right\|_{H^{s}}+C \int_{0}^{t}\left(\|m\|_{H^{\frac{1}{2}+\varepsilon_{0}}}\|n\|_{H^{\frac{1}{2}+\varepsilon_{0}}}\|m(\tau)\|_{H^{s}}+\|m\|_{H^{\frac{1}{2}+\varepsilon_{0}}}^{2}\|n(\tau)\|_{H^{s}}\right) \mathrm{d} \tau .
$$

For the second equation of the system (1.1), we can deal with it in a similar way and obtain that

$$
\|n\|_{H^{s}} \leq\left\|n_{0}\right\|_{H^{s}}+C \int_{0}^{t}\left(\|n\|_{H^{\frac{1}{2}+\varepsilon_{0}}}\|m\|_{H^{\frac{1}{2}+\varepsilon_{0}}}\|n(\tau)\|_{H^{s}}+\|n\|_{H^{\frac{1}{2}+\varepsilon_{0}}}^{2}\|m(\tau)\|_{H^{s}}\right) \mathrm{d} \tau .
$$

Hence, we have

$$
\begin{aligned}
& \|m\|_{H^{s}}+\|n\|_{H^{s}} \\
& \leq\left\|\mid m_{0}\right\|_{H^{s}}+\left\|n_{0}\right\|_{H^{s}}+C \int_{0}^{t}\left(\|n\|_{H^{\frac{1}{2}+\varepsilon_{0}}}+\|m\|_{H^{\frac{1}{2}+\varepsilon_{0}}}\right)^{2}\left(\|m(\tau)\|_{H^{s}}+\|n(\tau)\|_{H^{s}}\right) \mathrm{d} \tau .
\end{aligned}
$$

which implies the following results by the Gronwall's inequality

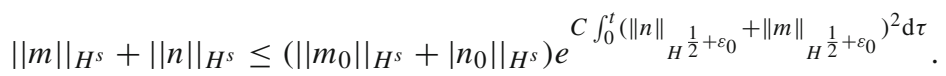

Therefore, if the maximal existence time $T<\infty$ satisfies $\int_{0}^{T}\left(\|n\|_{H^{\frac{1}{2}+\varepsilon_{0}}}+\|m\|_{H^{\frac{1}{2}+\varepsilon_{0}}}\right)^{2} \mathrm{~d} \tau<$ $\infty$, then we deduce from the uniqueness of the solution to the system (1.1) and (3.10) with 
$\frac{1}{2}+\varepsilon_{0} \in\left(\frac{1}{2}, 1\right)$ that $\|n\|_{H^{\frac{1}{2}+\varepsilon_{0}}}+\|m\|_{H^{\frac{1}{2}+\varepsilon_{0}}}$ is uniformly bounded in $t \in(0, T)$. This along with (3.15) implies that

$$
\limsup _{t \rightarrow T}\left(\|m(t)\|_{H^{s}}+\|n(t)\|_{H^{s}}\right)<\infty,
$$

which contradicts the assumption that $T<\infty$ is the maximal existence time. Thus, the theorem is also correct for $s \in\left[1, \frac{3}{2}\right)$.

Step 3. For $s \in(1,2)$, by differentiating the first equation in (2.1) with respect to $x$, we have

$$
\begin{aligned}
& \partial_{x} m_{t}+2 u v \partial_{x} m_{x}=\left(-4 u v_{x}-6 v u_{x}\right) m_{x}+\left(-2 u v_{x}-4 v u_{x}\right)_{x} m, \\
& \partial_{x} n_{t}+2 v u \partial_{x} n_{x}=\left(-4 v u_{x}-6 v u_{x}\right) n_{x}+\left(-2 v u_{x}-4 u v_{x}\right)_{x} m .
\end{aligned}
$$

By Lemma 2.4 with $s-1 \in(0,1)$, we get

$$
\begin{aligned}
\left\|\partial_{x} m(t)\right\|_{H^{s-1}} \leq & \left\|\partial_{x} m_{0}\right\|_{H^{s-1}}+C \int_{0}^{t}\left(\|u v\|_{L^{\infty}}+\left\|u_{x} v+u v_{x}\right\|_{L^{\infty}}\right) \partial_{x} m \|_{H^{s-1}} \mathrm{~d} \tau \\
& +C \int_{0}^{t}\left\|\left(-4 u v_{x}-6 v u_{x}\right) m_{x}+\left(-2 u v_{x}-4 v u_{x}\right)_{x} m\right\|_{H^{s-1}} \mathrm{~d} \tau
\end{aligned}
$$

and

$$
\begin{aligned}
\left\|\partial_{x} n(t)\right\|_{H^{s-1}} \leq & \left\|\partial_{x} n_{0}\right\|_{H^{s-1}}+C \int_{0}^{t}\left(\|u v\|_{L^{\infty}}+\left\|u_{x} v+u v_{x}\right\|_{L^{\infty}} \|\right) \partial_{x} n \|_{H^{s-1}} \mathrm{~d} \tau \\
& +C \int_{0}^{t}\left\|\left(-4 v u_{x}-6 u v_{x}\right) n_{x}+\left(-2 v u_{x}-4 u v_{x}\right)_{x} n\right\|_{H^{s-1}} \mathrm{~d} \tau
\end{aligned}
$$

Thanks to Lemma 2.5 and (3.2)-(3.3), we have

$$
\begin{aligned}
\left\|\left(-4 v u_{x}-6 u v_{x}\right) m_{x}\right\|_{H^{s-1}} \leq & C\left(\left\|\left(-4 v u_{x}-6 u v_{x}\right)\right\|_{H^{s}}\|m\|_{L^{\infty}}\right. \\
& \left.+\left\|\left(-4 v u_{x}-6 u v_{x}\right)\right\|_{L^{\infty}}\left\|m_{x}\right\|_{H^{s-1}}\right) \\
\leq & C\left(\|m\|_{L^{\infty}}\|n\|_{L^{\infty}}\|m\|_{H^{s}}+\|m\|_{L^{\infty}}^{2}\|n\|_{H^{s}}\right),
\end{aligned}
$$

and

$$
\begin{aligned}
& \left\|\left(-2 u v_{x}-4 v u_{x}\right)_{x} m\right\|_{H^{s-1}} \leq C\left(\left\|-2 u v_{x}-4 v u_{x}\right\|_{H^{s}}\|m\|_{L^{\infty}}\right. \\
& \left.+\left\|-2 u v_{x}-4 v u_{x}\right\|_{L^{\infty}}\|m\|_{H^{s}}\right) \\
& \leq C\left(\|m\|_{L^{\infty}}\|n\|_{L^{\infty}}\|m\|_{H^{s}}+\|m\|_{L^{\infty}}^{2}\|n\|_{H^{s}}\right),
\end{aligned}
$$

which together with (3.5) yields

$$
\left\|\partial_{x} m\right\|_{H^{s-1}} \leq\left\|m_{0}\right\|_{H^{s}}+C \int_{0}^{t}\left(\|m\|_{L^{\infty}}\|n\|_{L^{\infty}}\|m\|_{H^{s}}+\|m\|_{L^{\infty}}^{2}\|n\|_{H^{s}}\right) \mathrm{d} \tau .
$$

Similarly, we get

$$
\left\|\partial_{x} n\right\|_{H^{s-1}} \leq\left\|n_{0}\right\|_{H^{s}}+C \int_{0}^{t}\left(\|n\|_{L^{\infty}}\|m\|_{L^{\infty}}\|n\|_{H^{s}}+\|n\|_{L^{\infty}}^{2}\|m\|_{H^{s}}\right) \mathrm{d} \tau .
$$

Thus, we have

$$
\begin{aligned}
& \left\|\partial_{x} m\right\|_{H^{s-1}}+\left\|\partial_{x} n\right\|_{H^{s-1}} \\
& \leq\left\|m_{0}\right\|_{H^{s}}+\left\|n_{0}\right\|_{H^{s}}+C \int_{0}^{t}\left(\|m\|_{L^{\infty}}+\|n\|_{L^{\infty}}\right)^{2}\left(\|m\|_{H^{s}}+\|n\|_{H^{s}}\right) \mathrm{d} \tau .
\end{aligned}
$$


This along with (3.8) with $s-1$ instead of $s$ ensures

$$
\begin{aligned}
& \|m\|_{H^{s-1}}+\|n\|_{H^{s-1}} \\
& \leq\left\|m_{0}\right\|_{H^{s}}+\left\|n_{0}\right\|_{H^{s}}+C \int_{0}^{t}\left(\|m\|_{L^{\infty}}+\|n\|_{L^{\infty}}\right)^{2}\left(\|m\|_{H^{s}}+\|n\|_{H^{s}}\right) \mathrm{d} \tau .
\end{aligned}
$$

Similar to Step 1, we can easily prove the theorem for $s \in(1,2)$.

Step 4. For $s=k \in \mathbb{N}$ and $k \geq 2$, differentiating The system (1.1) $k-1$ times with respect to $x$ gives

$$
\begin{aligned}
& \partial_{x}^{k-1} m_{t}+2 u v \partial_{x}^{k-1} m_{x}=-2 \sum_{l=0}^{k-2} C_{k-1}^{l} \partial_{x}^{k-l-1}(u v) \partial_{x}^{l+1} m-\partial_{x}^{k-1}\left(2 u v_{x} m+4 v u_{x} m\right) \doteq F_{1}, \\
& \partial_{x}^{k-1} n_{t}+2 u v \partial_{x}^{k-1} n_{x}=-2 \sum_{l=0}^{k-2} C_{k-1}^{l} \partial_{x}^{k-l-1}(u v) \partial_{x}^{l+1} n-\partial_{x}^{k-1}\left(2 v u_{x} n+4 u v_{x} n\right) \doteq F_{2},
\end{aligned}
$$

which together with Lemma 2.3 imply

$$
\begin{aligned}
& \left\|\partial_{x}^{k-1} m(t)\right\|_{H^{1}} \leq\left\|m_{0}\right\|_{H^{k}}+\int_{0}^{t}\left\|F_{1}\right\|_{H^{1}} \mathrm{~d} \tau+C \int_{0}^{t}\left\|u_{x} v+u v_{x}\right\|_{H^{\frac{1}{2}} \cap L^{\infty}}\|m\|_{H^{k}} \mathrm{~d} \tau, \\
& \left\|\partial_{x}^{k-1} n(t)\right\|_{H^{1}} \leq\left\|n_{0}\right\|_{H^{k}}+\int_{0}^{t}\left\|F_{2}\right\|_{H^{1}} \mathrm{~d} \tau+C \int_{0}^{t}\left\|u_{x} v+u v_{x}\right\|_{H^{\frac{1}{2}} \cap L^{\infty}}\|n\|_{H^{k}} \mathrm{~d} \tau .
\end{aligned}
$$

Because of inequalities (3.2)-(3.3), we have

$$
\begin{aligned}
& \left\|-2 \sum_{l=0}^{k-2} C_{k-1}^{l} \partial_{x}^{k-l-1}(u v) \partial_{x}^{l+1} m\right\|_{H^{1}} \\
& \leq C(k)\left(\sum_{l=0}^{k-2}\left\|\partial_{x}^{k-l-1}(u v)\right\|_{L^{\infty}}\|m\|_{H^{l+2}}+\left\|\partial_{x}^{k-l-1}(u v)\right\|_{H^{1}}\left\|\partial_{x}^{l+1} m\right\|_{L^{\infty}}\right) \\
& \leq C(k) \sum_{l=0}^{k-2}\left(\left\|\partial_{x}^{k-l-1}(u v)\right\|_{H^{k-l-\frac{1}{2}+\varepsilon_{0}}}\|m\|_{H^{l+2}}+\|u v\|_{H^{k-l}}\|m\|_{H^{l+\frac{3}{2}+\varepsilon_{0}}}\right) \\
& \leq C(k)\left(\|u v\|_{H^{k-l-\frac{1}{2}+\varepsilon_{0}}}\|m\|_{H^{k}}+\|u v\|_{H^{k}}\|m\|_{H^{k-\frac{1}{2}+\varepsilon_{0}}}\right), \\
& \leq C(k)\left(\|m\|_{H^{k-\frac{1}{2}+\varepsilon_{0}}}\|n\|_{H^{k-\frac{1}{2}+\varepsilon_{0}}}\|m\|_{H^{k}}+\|m\|_{H^{k-\frac{1}{2}+\varepsilon_{0}}}^{2}\|n\|_{H^{k}}\right)
\end{aligned}
$$

and

$$
\begin{aligned}
& \left\|-\partial_{x}^{k-1}\left(2 u v_{x} m+4 v u_{x} m\right)\right\|_{H^{1}} \\
& \leq\left\|2 u v_{x} m+4 v u_{x} m\right\|_{H^{k}} \\
& \leq C\left(\|m\|_{H^{k-\frac{1}{2}+\varepsilon_{0}}}\|n\|_{H^{k-\frac{1}{2}+\varepsilon_{0}}}\|m\|_{H^{k}}+\|m\|_{H^{k-\frac{1}{2}+\varepsilon_{0}}}^{2}\|n\|_{H^{k}}\right),
\end{aligned}
$$

and

$$
\left\|u_{x} v+u v_{x}\right\|_{H^{\frac{1}{2}} \cap L^{\infty}} \leq C\left\|u_{x} v+u v_{x}\right\|_{H^{k-\frac{1}{2}+\varepsilon_{0}}} \leq C\|m\|_{H^{k-\frac{1}{2}+\varepsilon_{0}}}\|n\|_{H^{k-\frac{1}{2}+\varepsilon_{0}}},
$$

where $\varepsilon_{0} \in\left(0, \frac{1}{2}\right)$ and

$$
H^{k-\frac{1}{2}+\varepsilon_{0}}(\mathbb{R}) \hookrightarrow H^{\frac{1}{2}+\varepsilon_{0}}(\mathbb{R}) \hookrightarrow H^{\frac{1}{2}}(\mathbb{R}) \cap L^{\infty}(\mathbb{R}) \text { with } k \geq 2,
$$


is used in the above derivation. So, we obtain

$$
\begin{aligned}
& \left\|\partial_{x}^{k-1} m(t)\right\|_{H^{1}} \leq\left\|m_{0}\right\|_{H^{k}} \\
& \quad+C \int_{0}^{t}\left(\|m\|_{H^{k-\frac{1}{2}+\varepsilon_{0}}}\|n\|_{H^{k-\frac{1}{2}+\varepsilon_{0}}}\|m\|_{H^{k}}+\|m\|_{H^{k-\frac{1}{2}+\varepsilon_{0}}}^{2}\|n\|_{H^{k}}\right) \mathrm{d} \tau .
\end{aligned}
$$

Similarly, we get

$\left\|\partial_{x}^{k-1} n(t)\right\|_{H^{1}} \leq\left\|n_{0}\right\|_{H^{k}}+C \int_{0}^{t}\left(\|n\|_{H^{k-\frac{1}{2}+\varepsilon_{0}}}\|m\|_{H^{k-\frac{1}{2}+\varepsilon_{0}}}\|n\|_{H^{k}}+\|n\|_{H^{k-\frac{1}{2}+\varepsilon_{0}}}^{2}\|m\|_{H^{k}}\right) \mathrm{d} \tau$.

Thus, we have

$$
\begin{aligned}
& \left\|\partial_{x}^{k-1} m(t)\right\|_{H^{1}}+\left\|\partial_{x}^{k-1} n(t)\right\|_{H^{1}} \\
& \leq\left\|m_{0}\right\|_{H^{k}}+\left\|n_{0}\right\|_{H^{k}}+C \int_{0}^{t}\left(\|n\|_{H^{k-\frac{1}{2}+\varepsilon_{0}}}+\|m\|_{H^{k-\frac{1}{2}+\varepsilon_{0}}}\right)^{2}\left(\|m\|_{H^{k}}+\|n\|_{H^{k}}\right) \mathrm{d} \tau .
\end{aligned}
$$

Therefore, by the Gronwall's inequality and (3.15) with $s=1$, we have

$$
\|m\|_{H^{k}}+\|n\|_{H^{k}} \leq\left(\left\|m_{0}\right\|_{H^{k}}+\mid n_{0} \|_{H^{k}}\right) e^{C \int_{0}^{t}(\|n\|}{ }_{H^{k-\frac{1}{2}+\varepsilon_{0}}}+\|m\|{ }_{\left.k-H^{\frac{1}{2}+\varepsilon_{0}}\right)^{2} \mathrm{~d} \tau} .
$$

Therefore, if the maximal existence time $T<\infty$ satisfies $\int_{0}^{T}\left(\|n\|_{H^{\frac{1}{2}+\varepsilon_{0}}}+\|m\|_{H^{\frac{1}{2}+\varepsilon_{0}}}\right)^{2} \mathrm{~d} \tau<$ $\infty$, applying Step 3 with $\frac{3}{2}+\varepsilon_{0} \in(1,2)$ and by induction with respect to $k \geq 2$, we see that $\|n\|_{H^{k-\frac{1}{2}+\varepsilon_{0}}}+\|m\|_{H^{k-\frac{1}{2}+\varepsilon_{0}}}$ is uniformly bounded in $t \in(0, T)$. This along with (3.35) implies that

$$
\limsup _{t \rightarrow T}\left(\|m(t)\|_{H^{k}}+\|n(t)\|_{H^{k}}\right)<\infty
$$

which contradicts the assumption that $T<\infty$ is the maximal existence time. Thus, the theorem is also correct for $s=k \in \mathbb{N}$ and $k \geq 2$.

Step 5. For $s \in(k, k+1), k \in \mathbb{N}$ and $k \geq 2$, differentiating the system (1.1) $k$ times with respect to $x$ yields

$$
\begin{aligned}
& \partial_{x}^{k} m_{t}+2 u v \partial_{x}^{k} m_{x}=-2 \sum_{l=0}^{k-1} C_{k}^{l} \partial_{x}^{k-l}(u v) \partial_{x}^{l+1} m-\partial_{x}^{k}\left(2 u v_{x} m+4 v u_{x} m\right) \doteq G_{1}, \\
& \partial_{x}^{k} n_{t}+2 u v \partial_{x}^{k} n_{x}=-2 \sum_{l=0}^{k-1} C_{k}^{l} \partial_{x}^{k-l}(u v) \partial_{x}^{l+1} n-\partial_{x}^{k}\left(2 v u_{x} n+4 u v_{x} n\right) \doteq G_{2},
\end{aligned}
$$

which together with Lemma 2.3 as $s-k \in(0,1)$ imply

$$
\begin{aligned}
& \left\|\partial_{x}^{k} m(t)\right\|_{H^{s-k}} \leq\left\|\partial_{x}^{k} m_{0}\right\|_{H^{s-k}}+\int_{0}^{t}\left\|G_{1}\right\|_{H^{s-k}} \mathrm{~d} \tau+C \int_{0}^{t}\left\|u_{x} v+u v_{x}\right\|_{L^{\infty}}\left\|\partial_{x}^{k} m\right\|_{H^{s-k}} \mathrm{~d} \tau, \\
& \left\|\partial_{x}^{k} n(t)\right\|_{H^{s-k}} \leq\left\|\partial_{x}^{k} n_{0}\right\|_{H^{s-k}}+\int_{0}^{t}\left\|G_{2}\right\|_{H^{s-k}} \mathrm{~d} \tau+C \int_{0}^{t}\left\|u_{x} v+u v_{x}\right\|_{L^{\infty}}\left\|\partial_{x}^{k} n\right\|_{H^{s-k}} \mathrm{~d} \tau .
\end{aligned}
$$


By (3.30) and using the procedure similar to (3.28)-(3.30), we obtain

$\left\|\partial_{x}^{k} m(t)\right\|_{H^{s-k}} \leq\left\|m_{0}\right\|_{H^{k}}+C \int_{0}^{t}\left(\|m\|_{H^{k-\frac{1}{2}+\varepsilon_{0}}}\|n\|_{H^{k-\frac{1}{2}+\varepsilon_{0}}}\|m\|_{H^{s}}+\|m\|_{H^{k-\frac{1}{2}+\varepsilon_{0}}}^{2}\|n\|_{H^{s}}\right) \mathrm{d} \tau$,

and

$\left\|\partial_{x}^{k} n(t)\right\|_{H^{s-k}} \leq\left\|n_{0}\right\|_{H^{k}}+C \int_{0}^{t}\left(\|n\|_{H^{k-\frac{1}{2}+\varepsilon_{0}}}\|m\|_{H^{k-\frac{1}{2}+\varepsilon_{0}}}\|n\|_{H^{s}}+\|n\|_{H^{k-\frac{1}{2}+\varepsilon_{0}}}^{2}\|m\|_{H^{s}}\right) \mathrm{d} \tau$,

which imply

$$
\begin{aligned}
& \left\|\partial_{x}^{k} m(t)\right\|_{H^{s-k}}+\left\|\partial_{x}^{k} n(t)\right\|_{H^{s-k}}^{t} \\
& \leq\left\|m_{0}\right\|_{H^{s}}+\left\|n_{0}\right\|_{H^{s}}+C \int_{0}^{t}\left(\|n\|_{H^{k-\frac{1}{2}+\varepsilon_{0}}}+\|m\|_{H^{k-\frac{1}{2}+\varepsilon_{0}}}\right)^{2}\left(\|m\|_{H^{s}}+\|n\|_{H^{s}}\right) \mathrm{d} \tau .
\end{aligned}
$$

Therefore, by Gronwall's inequality and (3.15) with $s-k \in(0,1)$ and using the above inequality lead to

$$
\begin{aligned}
& \|m(t)\|_{H^{s}}+\|n(t)\|_{H^{s}} \\
& \leq\left\|m_{0}\right\|_{H^{s}}+\left\|n_{0}\right\|_{H^{s}}+C \int_{0}^{t}\left(\|n\|_{H^{k-\frac{1}{2}+\varepsilon_{0}}}+\|m\|_{H^{k-\frac{1}{2}+\varepsilon_{0}}}\right)^{2}\left(\|m\|_{H^{s}}+\|n\|_{H^{s}}\right) \mathrm{d} \tau .
\end{aligned}
$$

By adopting Gronwall's inequality, Step 3 with $\frac{3}{2}+\varepsilon_{0} \in(1,2)$, and the similar argument as shown in Step 4, we can arrive at the desired result.

In summary, the above 5 steps complete the proof of the theorem.

Now, we turn our attention to a blow-up scenario for the strong solution $(m, n)$ of the system (1.1). For this purpose, we need to consider the following ordinary differential equation:

$$
\begin{cases}\partial_{t} q(t, x)=2 u v(t, q(t, x)), & (t, x) \in(0, T) \times \mathbb{R} \\ q(0, x)=x, & x \in \mathbb{R}\end{cases}
$$

for the flow $q$ generated by $2 u v$.

The following lemmas are very crucial to the blow-up phenomena of strong solutions to the system (1.1)

Lemma 3.1 Let $\left(m_{0}, n_{0}\right) \in H^{s}(\mathbb{R}) \times H^{s}(\mathbb{R})\left(s>\frac{1}{2}\right)$ and $T>0$ be the maximal existence time of the corresponding solution $(m, n)$ to the system (1.1). Then Eq. (3.43) has a unique solution $q \in C^{1}([0, T) \times \mathbb{R} ; \mathbb{R})$. Moreover, the mapping $q(t, \cdot)$ is an increasing diffeomorphism of $\mathbb{R}$ with

$$
q_{x}(t, x)=\exp \left(2 \int_{0}^{t}\left(u_{x} v+u v_{x}\right)(s, q(s, x)) d s\right)>0
$$

for all $(t, x) \in[0, T) \times \mathbb{R}$.

Proof Since $(u, v) \in C\left([0, T) ; H^{s}(\mathbb{R}) \times H^{s}(\mathbb{R})\right) \cap C^{1}\left([0, T) ; H^{s-1}(\mathbb{R}) \times H^{s-1}(\mathbb{R})\right)$ as $s>\frac{1}{2}$, it follows from the fact $H^{s-1}(\mathbb{R}) \hookrightarrow \operatorname{Lip}(\mathbb{R})\left(s>\frac{5}{2}\right)$ that $2 u v$ is bounded and Lipschitz continuous in the space variable $x$ and of class $C^{1}$ in time variable $t$. Then the 
classical ODE theory ensures that Eq. (3.43) has a unique solution $q \in C^{1}([0, T) \times \mathbb{R} ; \mathbb{R})$ Differentiating Eq. (3.43) with respect to $x$ gives

$$
\begin{cases}\partial_{t} q_{x}(t, x)=2\left(u_{x} v+u v_{x}\right)(t, q(t, x)) q_{x}(t, x), & (t, x) \in(0, T) \times \mathbb{R}, \\ q_{x}(0, x)=1, & x \in \mathbb{R} .\end{cases}
$$

Solving the above ODE yields (3.44). Furthermore, it follows from the Sobolev embedding theorem, we have

$$
\sup _{(s, x) \in[0, T) \times \mathbb{R}}\left|2\left(u_{x} v+u v_{x}\right)(s, x)\right|<\infty .
$$

This along with (3.44) implies that there exists a constant $C>0$ such that

$$
q_{x}(t, x) \geq e^{-C t}, \quad \forall(t, x) \in[0, T) \times \mathbb{R} .
$$

This implies that $q(t, \cdot)$ is an increasing diffeomorphism of $\mathbb{R}$ before blow-up. This completes the proof of the lemma

Lemma 3.2 Let $\left(m_{0}, n_{0}\right) \in H^{s}(\mathbb{R}) \times H^{s}(\mathbb{R})\left(s>\frac{1}{2}\right)$ and $T>0$ be the maximal existence time of the solution $(m, n)$ corresponding to the system (1.1). Then, we have

$$
m(t, q(t, x)) q_{x}(t, x)=m_{0} \exp \left(2 \int_{0}^{t} u_{x} v(s, q(s, x)) \mathrm{d} x\right),
$$

and

$$
n(t, q(t, x)) q_{x}(t, x)=n_{0} \exp \left(-2 \int_{0}^{t} v_{x} u(s, q(s, x)) \mathrm{d} x\right),
$$

forall $(t, x) \in[0, T) \times \mathbb{R}$. Moreover, if there exists a $C>0$ such that $\left(u_{x} v+u v_{x}\right)(t, x) \geq-C$, $\left\|\left(u_{x} v\right)(t, \cdot)\right\|_{L^{\infty}} \leq C$ and $\left\|\left(u v_{x}\right)(t, \cdot)\right\|_{L^{\infty}} \leq C$ for all $(t, x) \in[0, T) \times \mathbb{R}$, then

$$
\|m(t, \cdot)\|_{L^{\infty}} \leq C e^{C t}\left\|m_{0}\right\|_{H^{s}} \text {, and }\|n(t, \cdot)\|_{L^{\infty}} \leq C e^{C t}\left\|n_{0}\right\|_{H^{s}},
$$

for all $t \in[0, T)$.

Proof Differentiating the left-hand side of (3.46)-(3.47) with respect to $t$ and making use of (3.33)-(3.34) and the system (1.1), we have

$$
\begin{aligned}
\frac{\mathrm{d}}{\mathrm{d} t}\left(m(t, q(t, x)) q_{x}(t, x)\right) & =\left(m_{t}(t, q)+m_{x}(t, x) q_{t}(t, x)\right) q_{x}(t, x)+m(t, q) q_{x t}(t, x) \\
& =\left(m_{t}+2 u v m_{x}+2\left(u_{x} v+u v_{x}\right) m\right)(t, q(t, x)) q_{x}(t, x) \\
& =-2 u_{x} v m(t, q(t, x)) q_{x}(t, x),
\end{aligned}
$$

and

$$
\begin{aligned}
\frac{\mathrm{d}}{\mathrm{d} t}\left(n(t, q(t, x)) q_{x}(t, x)\right) & =\left(n_{t}(t, q)+n_{x}(t, x) q_{t}(t, x)\right) q_{x}(t, x)+n(t, q) q_{x t}(t, x) \\
& =\left(n_{t}+2 u v n_{x}+2\left(u_{x} v+u v_{x}\right) n\right)(t, q(t, x)) q_{x}(t, x) \\
& =-2 v_{x} u n(t, q(t, x)) q_{x}(t, x),
\end{aligned}
$$

which guarantee (3.46) and (3.47). By Lemma 3.1, in light of (3.33)-(3.34) and the assumption, for all $t \in[0, T)$ we obtain

$$
\begin{aligned}
\|m(t, \cdot)\|_{L^{\infty}} & =\|m(t, q(t, \cdot))\|_{L^{\infty}} \\
& =\left\|e^{2 \int_{0}^{t} u_{x} v(s, q(s, x)) \mathrm{d} x} q_{x}^{-1}(t, \cdot)\right\|_{L^{\infty}}
\end{aligned}
$$




$$
\leq C e^{C t}\left\|m_{0}\right\|_{H^{s}}
$$

and

$$
\begin{aligned}
\|n(t, \cdot)\|_{L^{\infty}} & =\|n(t, q(t, \cdot))\|_{L^{\infty}} \\
& =\left\|e^{-2 \int_{0}^{t} v_{x} u(s, q(s, x)) \mathrm{d} x} q_{x}^{-1}(t, \cdot)\right\|_{L^{\infty}} \\
& \leq C e^{C t}\left\|n_{0}\right\|_{H^{s}},
\end{aligned}
$$

which complete the proof of the lemma.

The following theorem shows the precise blow-up scenario for sufficiently regular solutions to the system (1.1).

Theorem 3.2 Let $\left(m_{0}, n_{0}\right) \in H^{s}(\mathbb{R}) \times H^{s}(\mathbb{R})\left(s>\frac{1}{2}\right)$ and $T>0$ be the maximal existence time of the solution $(m, n)$ corresponding to the system $(1.1)$. Then the solution $(m, n)$ blows up in finite time if and only if

$$
\begin{aligned}
& \liminf _{t \rightarrow T} \inf _{x \in \mathbb{R}}\left\{\left(u_{x} v+u v_{x}\right)(t, x)\right\}=-\infty, \text { or } \\
& \limsup _{t \rightarrow T}\left(\left\|\left(u_{x} v\right)(t, \cdot)\right\|_{L^{\infty}}\right)=\infty, \text { or } \\
& \limsup _{t \rightarrow T}\left(\left\|\left(u v_{v}\right)(t, \cdot)\right\|_{L^{\infty}}\right)=\infty .
\end{aligned}
$$

Proof Assume that the solution $(m, n)$ blows up in finite time $(T<\infty)$ and there exists a constant $C>0$ such that $\left(u_{x} v+u v_{x}\right)(t, x) \geq-C,\left\|\left(u_{x} v\right)(t, \cdot)\right\|_{L^{\infty}} \leq C$ and $\left\|\left(u v_{x}\right)(t, \cdot)\right\|_{L^{\infty}} \leq C$ for all $(t, x) \in[0, T) \times \mathbb{R}$, By (3.48), we have

$$
\int_{x}^{T}\|m(t)\|_{L^{\infty}}\|n(t)\|_{L^{\infty}} \mathrm{d} t \leq C^{2} e^{2 C T}\left\|m_{0}\right\|_{H^{s}}\left\|n_{0}\right\|_{H^{s}}<\infty,
$$

which contradicts to Theorem 3.1.

On the other hand, by Sobolev's embedding theorem, we can see that if $\lim _{\inf } \rightarrow T \inf _{x \in \mathbb{R}}$ $\left\{\left(u_{x} v+u v_{x}\right)(t, x)\right\}=-\infty$, or $\limsup _{t \rightarrow T}\left(\left\|\left(u_{x} v\right)(t, \cdot)\right\|_{L^{\infty}}\right)=\infty$ or $\lim \sup _{t \rightarrow T}\left(\left\|\left(u v_{v}\right)(t, \cdot)\right\|_{L^{\infty}}\right)=\infty$ then the solution $(m, n)$ will blow up in finite time. Now, the proof of the theorem is completed.

\section{Analyticity of solutions}

In this section, we will show the existence and uniqueness of analytic solutions to the system (1.1) on the line $\mathbb{R}$.

First, we will need a suitable scale of Banach spaces as follows. For any $s>0$, we set

$$
E_{s}=\left\{u \in C^{\infty}(\mathbb{R}):\|\| u \mid \|_{s}=\sup _{k \in \mathbb{N}_{0}} \frac{s^{k}\left\|\partial^{k} u\right\|_{H^{2}}}{k ! /(k+1)^{2}}<\infty\right\},
$$

where $H^{2}(\mathbb{R})$ is the Sobolev space of order two on the real line $\mathbb{R}$ and $\mathbb{N}_{0}$ is the set of nonnegative integers. One can easily verify that $E_{S}$ equipped with the norm \|\|$\cdot\|\|_{s}$ is a Banach space and that, for any $0<s^{\prime}<s, E_{s}$ is continuously embedded in $E_{s^{\prime}}$ with

$$
\|\| u\|\|_{s^{\prime}} \leq\|\| u \|_{s} .
$$


Another simple consequence of the definition is that any $u$ in $E_{s}$ is a real analytic function on $\mathbb{R}$. Crucial for our purposes is the fact that each $E_{s}$ forms an algebra under pointwise multiplication of functions.

Lemma 4.1 [31] Let $0<s<1$. There is a constant $C>0$, independent of $s$, such that for any $u$ and $v$ in $E_{s}$ we have

$$
\|\| u v\left\|_{s} \leq C\right\||| u\left\|_{s}\right\|\|v\|_{s} .
$$

Lemma 4.2 [31] There is a constant $c>0$ such that for any $0<s^{\prime}<s<1$, we have \|\|$\partial_{x} u\|\|_{s^{\prime}} \leq \frac{C}{s-s^{\prime}} \mid\|u\|\left\|_{s},\right\|\left\|\left(1-\partial_{x}^{2}\right)^{-1} u\right\|\left\|_{s^{\prime}} \leq\right\|\|u\| \|_{s}$ and \|\|$\left(1-\partial_{x}^{2}\right)^{-1} \partial_{x} u\|\|_{s^{\prime}} \leq\|\| u\|\|_{s}$.

Theorem 4.1 [1] Let $\left\{X_{s}\right\}_{0<s<1}$ be a scale of decreasing Banach spaces, namely for any $s^{\prime}<s$ we have $X_{s} \subset X_{s^{\prime}}$ and $\|\mid \cdot\|\left\|_{s^{\prime}} \leq\right\|\|\cdot\| \|_{s}$. Consider the Cauchy problem

$$
\left\{\begin{array}{l}
\frac{\mathrm{d} u}{\mathrm{~d} t}=F(t, u(t)) \\
u(0)=0
\end{array}\right.
$$

Let $T, H$ and $C$ be positive constants and assume that $F$ satisfies the following conditions

(1) If for $0<s^{\prime}<s<1$ the function $t \mapsto u(t)$ is holomorphic in $|t|<T$ and continuous on $|t| \leq T$ with values in $X_{s}$ and

$$
\sup _{|t| \leq T}\|\| u(t)\|\|_{s}<H
$$

then $t \mapsto F(t, u(t))$ is a holomorphic function on $|t|<T$ with values in $X_{s^{\prime}}$.

(2) For any $0<s^{\prime}<s<1$ and any $u, v \in B(0, H) \subset X_{s}$, that is, \|\|$u\|\|_{s}<H,\|\| v\|\|_{s}<$ $H$, we have

$$
\sup _{|t| \leq T}\left|\|F(t, u)-F(t, v)\|\left\|_{s^{\prime}} \leq \frac{C}{s-s^{\prime}} \mid\right\| u-v\|\|_{s} .\right.
$$

(3) There exists $M>0$ such that for any $0<s<1$,

$$
\sup _{|t| \leq T}|||F(t, 0)|\|\|_{s} \leq \frac{M}{1-s} .
$$

Then there exists a $T_{0} \in(0, T)$ and a unique function $u(t)$, which for every $s \in(0,1)$ is holomorphic in $|t|<(1-s) T_{0}$ with values in $X_{s}$, and is a solution to the Cauchy problem (6.1).

We are now ready to state the third main result of the paper.

Theorem 4.2 If the initial data $\left(\begin{array}{c}u_{0} \\ v_{0}\end{array}\right)$ is real analytic on the line $\mathbb{R}$ and belongs in a space $E_{s_{0}}$, for some $0<s_{0} \leq 1$, then there exists an $\varepsilon>0$ and a unique solution $\left(\begin{array}{c}u \\ v\end{array}\right)$ to the Cauchy problem (1.1) that is analytic on $(-\varepsilon, \varepsilon) \times \mathbb{R}$.

We restate the Cauchy problem (1.1) in a more convenient form, we can rewrite the Cauchy problem (1.1) as follows

$$
\left\{\begin{array}{l}
u_{t}+2 u v u_{x}=-\left(1-\partial_{x}^{2}\right)^{-1}\left(4 u u_{x} v+2 u^{2} v_{x}\right. \\
\left.+u_{x}^{2} v_{x}\right)-2 \partial_{x}\left(1-\partial_{x}^{2}\right)^{-1}\left(u_{x}^{2} v+u_{x} u v_{x}\right) \\
v_{t}+2 u v v_{x}=-\left(1-\partial_{x}^{2}\right)^{-1}\left(4 v v_{x} u+2 v^{2} u_{x}\right. \\
\left.+v_{x}^{2} u_{x}\right)-2 \partial_{x}\left(1-\partial_{x}^{2}\right)^{-1}\left(v_{x}^{2} u+v_{x} v u_{x}\right), \\
u(0, x)=u_{0}(x), v(0, t)=v_{0}(x) .
\end{array}\right.
$$


Differentiating with respect to $x$ on both sides of the above equation and letting $u_{1}=u, u_{2}=$ $u_{x}, u_{3}=v$ and $u_{4}=v_{x}$, then the problem (3.3) can be written as a system for $u_{1}, u_{2}, u_{2}, u_{4}$

$$
\left\{\begin{array}{c}
\partial_{t} u_{1}=-2 u_{1} u_{3} u_{2}-\left(1-\partial_{x}^{2}\right)^{-1}\left(4 u_{1} u_{2} u_{3}+2 u_{1}^{2} u_{4}+u_{2}^{2} u_{4}\right) \\
-2 \partial_{x}\left(1-\partial_{x}^{2}\right)^{-1}\left(u_{2}^{2} u_{3}+u_{2} u_{1} u_{4}\right)=F_{1}\left(u_{1}, u_{2}, u_{3}, u_{4}\right), \\
\partial_{t} u_{2}=-2 u_{1} u_{3} \partial_{x} u_{2}-\partial_{x}\left(1-\partial_{x}^{2}\right)^{-1}\left(4 u_{1} u_{2} u_{3}+2 u_{1}^{2} u_{4}+u_{2}^{2} u_{4}\right) \\
\quad-2\left(1-\partial_{x}^{2}\right)^{-1}\left(u_{2}^{2} u_{3}+u_{2} u_{1} u_{4}\right)=F_{1}\left(u_{1}, u_{2}, u_{3}, u_{4}\right) \\
\partial_{t} u_{3}=-2 u_{1} u_{3} u_{4}-\left(1-\partial_{x}^{2}\right)^{-1}\left(4 u_{3} u_{4} u_{1}+2 u_{3}^{2} u_{2}+u_{4}^{2} u_{2}\right) \\
\quad-2 \partial_{x}\left(1-\partial_{x}^{2}\right)^{-1}\left(u_{4}^{2} u_{1}+u_{4} u_{1} u_{2}\right)=F_{1}\left(u_{1}, u_{2}, u_{3}, u_{4}\right), \\
\partial_{t} u_{4}=-2 u_{1} u_{3} \partial_{x} u_{4}-\partial_{x}\left(1-\partial_{x}^{2}\right)^{-1}\left(4 u_{1} u_{4} u_{1}+2 u_{3}^{2} u_{2}+u_{4}^{2} u_{2}\right) \\
\quad-2\left(1-\partial_{x}^{2}\right)^{-1}\left(u_{4}^{2} u_{1}+u_{4} u_{3} u_{2}\right)=F_{1}\left(u_{1}, u_{2}, u_{3}, u_{4}\right) \\
u_{1}(0, x)=u_{0}(x) \\
u_{2}(0, x)=u_{0}^{\prime}(x) \\
u_{3}(0, x)=v_{0}(x) \\
u_{4}(0, x)=v_{0}^{\prime}(x)
\end{array}\right.
$$

Define

$$
U \equiv\left(u_{1}, u_{2}, u_{3}, u_{4}\right)
$$

and

$$
\begin{aligned}
F(U) & =F\left(u_{1}, u_{2}, u_{3}, u_{4}\right) \\
& \equiv\left(F_{1}\left(u_{1}, u_{2}, u_{3}, u_{4}\right), F_{2}\left(u_{1}, u_{2}, u_{3}, u_{4}\right), F_{3}\left(u_{1}, u_{2}, u_{3}, u_{4}\right), F_{4}\left(u_{1}, u_{2}, u_{3}, u_{4}\right)\right) .
\end{aligned}
$$

Then we have

$$
\left\{\begin{array}{l}
\frac{\mathrm{d} u}{\mathrm{~d} t}=F(t, U(t)), \\
U(0)=\left(u_{0}, u_{0}^{\prime}, v_{0}, v_{0}^{\prime}\right) .
\end{array}\right.
$$

Proof Theorem 4.2 is a straightforward consequence of the abstract Cauchy-Kowalevski theorem 4.1. We only need to verify the conditions (1)-(2) in the statement of the abstract Cauchy-Kowalevski Theorem 4.1 for $F_{i}\left(u_{1}, u_{2}, u_{3}, u_{4}\right),(i=1,2,3,4)$ in the system (4.3) since $F_{i}\left(u_{1}, u_{2}, u_{3}, u_{4}\right),(i=1,2,3,4)$ does not depend on $t$ explicitly. We observe that, for $0<s^{\prime}<s<1$, by the estimates in Lemmas 4.1 and 4.2, that condition (1) holds

Next, we verify the second condition. For any $u_{j}$ and $v_{j} \in B(0, H) \subset E_{S},(j=1,2,3,4)$, we have

$$
\begin{aligned}
& \|\| F\left(u_{1}, u_{2}, u_{3}, u_{4}\right)-F\left(v_{1}, v_{2}, v_{3}, v_{4}\right)\|\|_{s^{\prime}} \\
& \quad=\sum_{i=1}^{4}\left\||| F_{i}\left(u_{1}, u_{2}, u_{3}, u_{4}\right)-F_{i}\left(v_{1}, v_{2}, v_{3}, v_{4}\right)\right\| \|_{s^{\prime}} \\
& \quad \equiv I_{1}+I_{2}+I_{3}+I_{4} .
\end{aligned}
$$

We will estimate $I_{1}, I_{2}, I_{3}$ and $I_{4}$, respectively,

$$
\begin{aligned}
& I_{1} \leq 2||\left|u_{1} u_{3} u_{2}-v_{1} v_{3} v_{2}\right|||_{s^{\prime}}+||\left|\left(1-\partial_{x}^{2}\right)^{-1}\left(4 u_{1} u_{2} u_{3}-4 v_{1} v_{2} v_{3}\right)\right|||_{s^{\prime}} \\
& +||\left|\left(1-\partial_{x}^{2}\right)^{-1}\left(2 u_{1}^{2} u_{4}-2 v_{1}^{2} v_{4}\right)\right|||_{s^{\prime}}+||\left|\left(1-\partial_{x}^{2}\right)^{-1}\left(u_{2}^{2} u_{4}-v_{2}^{2} v_{4}\right)\right|||_{s^{\prime}} \\
& +\left\|\left|\partial_{x}\left(1-\partial_{x}^{2}\right)^{-1}\left(u_{2}^{2} u_{3}-v_{2}^{2} v_{3}\right)\left\|\left.\right|_{s^{\prime}}+\right\|\right| \partial_{x}\left(1-\partial_{x}^{2}\right)^{-1}\left(u_{2} u_{1} u_{4}-v_{2} v_{1} v_{4}\right)\right\| \|_{s^{\prime}} \\
& \leq 6||\left|u_{1} u_{3} u_{2}-v_{1} v_{3} v_{2}\right|||_{s^{\prime}}+||\left|2 u_{1}^{2} u_{4}-2 v_{1}^{2} v_{4}\right|||_{s}+||\left|u_{2}^{2} u_{4}-v_{2}^{2} v_{4}\right|||_{s} \\
& +||\left|u_{2}^{2} u_{3}-v_{2}^{2} v_{3}\right|||_{s}+||\left|u_{2} u_{1} u_{4}-v_{2} v_{1} v_{4}\right|||_{s}
\end{aligned}
$$




$$
\begin{aligned}
& \leq C\left(\left.||\left|u_{1}\right|||_{s}||\left|u_{2}\right|||_{s}||\left|u_{3}-v_{3}\right|\right|_{s}+||\left|u_{1}\right|||_{s}||\left|v_{3}\right|||_{s}||\left|u_{2}-v_{2}\right|||_{s}\right. \\
& +||\left|v_{3}\right|||_{s}||\left|v_{2}\right|||_{s}||\left|u_{1}-v_{2}\right|||_{s}+\left.||\left|u_{1}\right|||_{s}^{2}||\left|u_{4}-v_{4}\right|\right|_{s} \\
& +||\left|u_{2}\right|||_{s}^{2}||\left|u_{4}-v_{4}\right|||_{s}+||\left|v_{4}\right|||_{s}^{2}||\left|u_{2}+v_{2}\right|||_{s}||\left|u_{2}-v_{2}\right|||_{s} \\
& +||\left|u_{2}\right|||_{s}^{2}||\left|u_{3}-v_{3}\right|||_{s}+||\left|v_{3}\right|||_{s}^{2}||\left|u_{2}+v_{2}\right|||_{s}||\left|u_{2}-v_{2}\right|||_{s} \\
& +||\left|u_{1}\right|||_{s}||\left|u_{2}\right|||_{s}||\left|u_{4}-v_{4}\right|||_{s}+||\left|u_{1}\right|||_{s}||\left|v_{4}\right|||_{s}||\left|u_{2}-v_{2}\right|||_{s} \\
& \left.+||\left|v_{4}\right|||_{s}||\left|v_{2}\right|||_{s}||\left|u_{1}-v_{2}\right|||_{s}+||\left|v_{4}\right|||_{s}^{2}||\left|u_{1}+v_{1}\right|||_{s}||\left|u_{1}-v_{1}\right|||_{s}\right) \\
& \leq \frac{C}{s-s^{\prime}} \mid\left\|\left(u_{1}, u_{2}, u_{3}, u_{4}\right)-\left(u_{1}, u_{2}, u_{3}, u_{4}\right)\right\| \|_{s} \text {. }
\end{aligned}
$$

and

$$
\begin{aligned}
& I_{2} \leq 2||\left|u_{1} u_{3} \partial_{x} u_{2}-v_{1} v_{3} \partial_{x} v_{2}\right|||_{s^{\prime}}+\|\left.||\left(1-\partial_{x}^{2}\right)^{-1}\left(4 u_{1} u_{2} u_{3}-4 v_{1} v_{2} v_{3}\right)||\right|_{s^{\prime}} \\
& +||\left|\left(1-\partial_{x}^{2}\right)^{-1}\left(2 u_{1}^{2} u_{4}-2 v_{1}^{2} v_{4}\right)\left\|\left.\right|_{s^{\prime}}+\right\|\right| \mid\left(1-\partial_{x}^{2}\right)^{-1}\left(u_{2}^{2} u_{4}-v_{2}^{2} v_{4}\right)\|\|_{s^{\prime}} \\
& +\left\|\left|\partial_{x}\left(1-\partial_{x}^{2}\right)^{-1}\left(u_{2}^{2} u_{3}-v_{2}^{2} v_{3}\right)\right|\right\|_{s^{\prime}}+\left\|\mid \partial_{x}\left(1-\partial_{x}^{2}\right)^{-1}\left(u_{2} u_{1} u_{4}-v_{2} v_{1} v_{4}\right)\right\| \|_{s^{\prime}} \\
& \leq 6||\left|u_{1} u_{3} u_{2}-v_{1} v_{3} v_{2}\right|||_{s^{\prime}}+||\left|2 u_{1}^{2} u_{4}-2 v_{1}^{2} v_{4}\right|||_{s}+||\left|u_{2}^{2} u_{4}-v_{2}^{2} v_{4}\right|||_{s} \\
& +||\left|u_{2}^{2} u_{3}-v_{2}^{2} v_{3}\right|||_{s}+||\left|u_{2} u_{1} u_{4}-v_{2} v_{1} v_{4} \|_{\mid}\right|_{s} \\
& \leq C\left(||\left|u_{1}\right|||_{s}||\left|u_{2}\right|||_{s}|| u_{3}-v_{3}||_{s}+||\left|u_{1}\right|||_{s}||\left|v_{3}\right|||_{s}||\left|u_{2}-v_{2}\right| \|_{s}\right. \\
& +||\left|v_{3}\right|||_{s}||\left|v_{2}\right|||_{s}||\left|u_{1}-v_{2}\right|||_{s}+||\left|u_{1}\right|||_{s}^{2}||\left|u_{4}-v_{4}\right|||_{s} \\
& +||\left|u_{2}\right|||_{s}^{2}||\left|u_{4}-v_{4}\right|||_{s}+||\left|v_{4}\right|||_{s}^{2}||\left|u_{2}+v_{2}\right|||_{s}||\left|u_{2}-v_{2}\right|||_{s} \\
& +||\left|u_{2}\right|||_{s}^{2}||\left|u_{3}-v_{3}\right|||_{s}+||\left|v_{3}\right|||_{s}^{2}||\left|u_{2}+v_{2}\right|||_{s}||\left|u_{2}-v_{2}\right|||_{s} \\
& +||\left|u_{1}\right|||_{s}||\left|u_{2}\right|||_{s}||\left|u_{4}-v_{4}\right|||_{s}+||\left|u_{1}\right|||_{s}||\left|v_{4}\right|||_{s}||\left|u_{2}-v_{2}\right|||_{s} \\
& +||\left|v_{4}\right|||_{s}||\left|v_{2}\right|||_{s}||\left|u_{1}-v_{2}\right|||_{s}+||\left|v_{4}\right|||_{s}^{2}||\left|u_{1}+v_{1}\right|||_{s} \\
& +\frac{C}{s-s^{\prime}}\left(||\left|u_{1}-v_{1}\right|||_{s}||\left|u_{1}\right|||_{s}||\left|u_{2}\right|||_{s}||\left|u_{3}-v_{3}\right|||_{s}+||\left|u_{1}\right|||_{s}||\left|v_{3}\right|||_{s}||\left|u_{2}-v_{2}\right|||_{s}\right. \\
& \left.\left.+||\left|v_{3}\right|||_{s}||\left|v_{2}\right|||_{s}||\left|u_{1}-v_{2}\right|||_{s}\right)\right) \\
& \leq \frac{C}{s-s^{\prime}}\left\|\left(u_{1}, u_{2}, u_{3}, u_{4}\right)-\left(u_{1}, u_{2}, u_{3}, u_{4}\right)\right\|_{s} \text {. }
\end{aligned}
$$

In a similar way to what we just did, we can show that the following estimates hold

$$
\begin{aligned}
& I_{3} \leq \frac{C}{s-s^{\prime}}\left\|\mid\left(u_{1}, u_{2}, u_{3}, u_{4}\right)-\left(u_{1}, u_{2}, u_{3}, u_{4}\right)\right\| \|_{s}, \\
& I_{4} \leq \frac{C}{s-s^{\prime}}\left\|\mid\left(u_{1}, u_{2}, u_{3}, u_{4}\right)-\left(u_{1}, u_{2}, u_{3}, u_{4}\right)\right\| \|_{s},
\end{aligned}
$$

where the constant $C$ depends only on $H$. This implies that the condition (2) also holds. The conditions (1) through (3) above are now easily verified once our system (4.3) is transformed into a new system with zero initial data as in (4.1). The proof of Theorem 4.2 is complete.

\section{Persistence properties}

In this section, we present two persistence properties based on the work for $\mathrm{CH}$ equation $[6,29,30,35,53]$. For our convenience, we rewrite the system (1.1) as the form of a quasilinear evolution equation of hyperbolic type. Note that $G(x) \triangleq \frac{1}{2} e^{-|x|}$ is the kernel of 
$\left(1-\partial_{x}^{2}\right)^{-1}$. Then $\left(1-\partial_{x}^{2}\right)^{-1} f=G * f$ for all $f \in L^{2}(\mathbb{R}), G * m=u$ and $G * n=v$. By these identities, the system (1.1)) can be reformulated as follows

$$
\left\{\begin{array}{l}
u_{t}+2 u v u_{x}=G * F_{1}+\partial_{x} G * F_{2}, t>0, x \in \mathbb{R}, \\
n_{t}+2 u v n_{x}=G * H_{1}+\partial_{x} G * H_{2}, t>0, x \in \mathbb{R}, \\
u(0, x)=u_{0}(x), v(0, t)=v_{0}(x), \quad x \in \mathbb{R} .
\end{array}\right.
$$

where $M=u_{x} v+u v_{x}, F_{1}=-2\left(u M+u_{x} v m\right), F_{2}=-2 u_{x} M, H_{1}=-2(v M+$ $\left.v_{x} u n\right), H_{2}=-2 v_{x} M$.

Assume that $z \in C(0 ; T) ; H^{s} \times H^{s}$ is a strong solution to (1.1) with $s \geq 3$. Let

$$
K=\sup _{t \in[0, T]}\|z(t)\|_{H^{s}}=\sup _{t \in[0, T]}\left(\|u(t)\|_{H^{s}}+\|v(t)\|_{H^{s}}\right),
$$

hence by Sobolev imbedding theorem, we have

$$
\begin{gathered}
\|u(t, \cdot)\|_{L^{\infty}}+\left\|u_{x}(t, \cdot)\right\|_{L^{\infty}}+\left\|u_{x x}(t, \cdot)\right\|_{L^{\infty}} \leq C K, \\
\|v(t, \cdot)\|_{L^{\infty}}+\left\|v_{x}(t, \cdot)\right\|_{L^{\infty}}+\left\|v_{x x}(t, \cdot)\right\|_{L^{\infty}} \leq C K .
\end{gathered}
$$

We are now ready to state the fourth main result of the paper.

Theorem 5.1 Assume that $s \geq 3, T>0$, and $z \in C\left([0 ; T] ; H^{s} \times H^{s}\right)$ is a solution of (1.1). If the initial data $z_{0}(x)=z(0, x)$ decays at infinity, more precisely, if there is some $\theta \in(0,1)$ such that as $|x| \rightarrow \infty$

$$
\begin{gathered}
\left|u_{0}(x)\right| \sim O\left(e^{-\theta|x|}\right),\left|u_{0 x}(x)\right| \sim O\left(e^{-\theta|x|}\right), \\
\left|v_{0}(x)\right| \sim O\left(e^{-\theta|x|}\right),\left|v_{0 x}(x)\right| \sim O\left(e^{-\theta|x|}\right),
\end{gathered}
$$

then as $|x| \rightarrow \infty$, we have

$$
\begin{aligned}
&|u(t, x)| \sim O\left(e^{-\theta|x|}\right),\left|u_{x}(t, x)\right| \sim O\left(e^{-\theta|x|}\right), \\
&|v(t, x)| \sim O\left(e^{-\theta|x|}\right),\left|v_{x}(t, x)\right| \sim O\left(e^{-\theta|x|}\right),
\end{aligned}
$$

uniformly with respect to $t \in[0, T]$.

Proof Set

$$
\Phi_{N}(x)= \begin{cases}e^{\theta|x|}, & |x|<N \\ e^{N|x|}, & |x| \geq N\end{cases}
$$

where $N \in \mathbb{N}$ and $\theta \in(0,1)$. Observe that for all $\mathbb{N}$ we have

$$
0 \leq\left|\Phi_{N}^{\prime}\right| \leq \Phi_{N}(x), \quad \text { a.e. } x \in \mathbb{R} .
$$

Multiplying the first equation of the system (5.1) by $\left(u \Phi_{N}\right)^{2 q-1} \Phi_{N}$ for $q \in N$ and integrating over the real line, we obtain

$$
\begin{aligned}
\frac{1}{2 q} \frac{1}{\mathrm{~d} t} \int\left(u \Phi_{N}\right)^{2 q} \mathrm{~d} x= & -2 \int u v u_{x}\left(u \Phi_{N}\right)^{2 q-1} \Phi_{N} \mathrm{~d} x+\int \partial_{x}\left(G * F_{2}\right)\left(u \Phi_{N}\right)^{2 q-1} \Phi_{N} \mathrm{~d} x \\
& +\int\left(G * F_{1}\right)\left(u \Phi_{N}\right)^{2 q-1} \Phi_{N} \mathrm{~d} x
\end{aligned}
$$

(5.2)-(5.3) and Holder's inequality lead us to achieve the following estimates

$$
\left|-2 \int u v u_{x}\left(u \Phi_{N}\right)^{2 q-1} \Phi_{N} \mathrm{~d} x\right| \leq C K^{2}\left\|u \Phi_{N}\right\|_{2 q}^{2 q-1}\left\|u_{x} \Phi_{N}\right\|_{2 q},
$$




$$
\left|\int\left(G * F_{1}\right)\left(u \Phi_{N}\right)^{2 q-1} \Phi_{N} \mathrm{~d} x\right| \leq\left\|u \Phi_{N}\right\|_{2 q}^{2 q-1}\left\|\left(G * F_{1}\right) \Phi_{N}\right\|_{2 q},
$$

and

$$
\left|\int \partial_{x}\left(G * F_{2}\right)\left(u \Phi_{N}\right)^{2 q-1} \Phi_{N} \mathrm{~d} x\right| \leq\left\|u \Phi_{N}\right\|_{2 q}^{2 q-1}\left\|\partial_{x}\left(G * F_{2}\right) \Phi_{N}\right\|_{2 q},
$$

From (5.6) and the above estimates, this implies

$$
\frac{\mathrm{d}}{\mathrm{d} t}\left\|u \Phi_{N}\right\|_{2 q} \leq C K^{2}\left\|u \Phi_{N}\right\|_{2 q}+\left\|\left(G * F_{1}\right) \Phi_{N}\right\|_{2 q}+\left\|\partial_{x}\left(G * F_{2}\right) \Phi_{N}\right\|_{2 q} .
$$

By Gronwall's inequality, (5.10) implies the following estimate

$$
\left\|u \Phi_{N}\right\|_{2 q} \leq e^{C K^{2} t}\left(\left\|u_{0} \Phi_{N}\right\|_{2 q}+\int_{0}^{t}\left(\left\|\left(G * F_{1}\right) \Phi_{N}\right\|_{2 q}+\left\|\partial_{x}\left(G * F_{2}\right) \Phi_{N}\right\|_{2 q}\right) \mathrm{d} \tau\right) .
$$

Now differentiating the first equation of the system (5.1) with respect to the spacial variable $x$, multiplying by $\left(u_{x} \Phi_{N}\right)^{2 q-1} \Phi_{N}$ and integrating over the real line yields

$$
\begin{aligned}
& \frac{1}{2 q} \frac{1}{\mathrm{~d} t} \int\left(u_{x} \Phi_{N}\right)^{2 q} \mathrm{~d} x \\
& =-2 \int u v u_{x x}\left(u_{x} \Phi_{N}\right)^{2 q-1} \Phi_{N} \mathrm{~d} x+\int \partial_{x}^{2}\left(G * F_{2}\right)\left(u_{x} \Phi_{N}\right)^{2 q-1} \Phi_{N} \mathrm{~d} x \\
& \quad+\int \partial_{x}\left(G * F_{1}\right)\left(u_{x} \Phi_{N}\right)^{2 q-1} \Phi_{N} \mathrm{~d}-2 \int M u_{x}\left(u_{x} \Phi_{N}\right)^{2 q-1} \Phi_{N} \mathrm{~d} x
\end{aligned}
$$

This leads us to obtain the following estimates

$$
\begin{gathered}
\left|\int \partial_{x}^{2}\left(G * F_{2}\right)\left(u_{x} \Phi_{N}\right)^{2 q-1} \Phi_{N} \mathrm{~d} x\right| \leq\left\|u_{x} \Phi_{N}\right\|_{2 q}^{2 q-1}\left\|\partial_{x}^{2}\left(G * F_{2}\right) \Phi_{N}\right\|_{2 q}, \\
\left|\int\left(\partial_{x} G * F_{1}\right)\left(u_{x} \Phi_{N}\right)^{2 q-1} \Phi_{N} \mathrm{~d} x\right| \leq\left\|u_{x} \Phi_{N}\right\|_{2 q}^{2 q-1}\left\|\left(\partial_{x} G * F_{1}\right) \Phi_{N}\right\|_{2 q}, \\
\left|-2 \int M u_{x}\left(u_{x} \Phi_{N}\right)^{2 q-1} \Phi_{N} \mathrm{~d} x\right| \leq\|M\|_{L^{\infty}}\left\|u_{x} \Phi_{N}\right\|_{2 q}^{2 q} \leq C K^{2}\left\|u_{x} \Phi_{N}\right\|_{2 q}^{2 q},
\end{gathered}
$$

and

$$
\begin{aligned}
\int u v u_{x x}\left(u_{x} \Phi_{N}\right)^{2 q-1} \Phi_{N} \mathrm{~d} x= & \int u v\left[\left(u_{x} \Phi_{N}\right)_{x}-u_{x} \Phi_{N}^{\prime}\right]\left(u_{x} \Phi_{N}\right)^{2 q-1} \Phi_{N} \mathrm{~d} x, \\
& -\frac{1}{2 q} \int M\left(u_{x} \Phi_{N}\right)^{2 q} \mathrm{~d} x-\int u v u_{x} \Phi_{N}^{\prime}\left(u_{x} \Phi_{N}\right)^{2 q-1} \mathrm{~d} x \\
\leq & C K^{2}\left\|u_{x} \Phi_{N}\right\|_{2 q}^{2 q} .
\end{aligned}
$$

From (5.12) to (5.14), we achieve the following differential inequality

$$
\frac{\mathrm{d}}{\mathrm{d} t}\left\|u_{x} \Phi_{N}\right\|_{2 q} \leq C K^{2}\left\|u_{x} \Phi_{N}\right\|_{2 q}+\left\|\left(\partial_{x}^{2} G * F_{2}\right) \Phi_{N}\right\|_{2 q}+\left\|\partial_{x}\left(G * F_{1}\right) \Phi_{N}\right\|_{2 q} .
$$

By Gronwall's inequality, (5.15) implies the following estimate

$$
\left\|u_{x} \Phi_{N}\right\|_{2 q} \leq e^{C K^{2} t}\left(\left\|u_{x 0} \Phi_{N}\right\|_{2 q}+\int_{0}^{t}\left(\left\|\left(\partial_{x} G * F_{1}\right) \Phi_{N}\right\|_{2 q}+\left\|\partial_{x}^{2}\left(G * F_{2}\right) \Phi_{N}\right\|_{2 q}\right) \mathrm{d} \tau\right) \text {. }
$$


By adding (5.11) and (5.16), we have the following

$$
\begin{aligned}
\left\|u \Phi_{N}\right\|_{2 q}+\left\|u_{x} \Phi_{N}\right\|_{2 q} \leq e^{C K^{2} t}\left(\left\|u_{0} \Phi_{N}\right\|_{2 q}+\left\|u_{x 0} \Phi_{N}\right\|_{2 q}\right. \\
+\int_{0}^{t}\left(\left\|\left(G * F_{1}\right) \Phi_{N}\right\|_{2 q}+\left\|\partial_{x}\left(G * F_{2}\right) \Phi_{N}\right\|_{2 q}\right) \mathrm{d} \tau \\
\left.+\int_{0}^{t}\left(\left\|\left(\partial_{x} G * F_{1}\right) \Phi_{N}\right\|_{2 q}+\left\|\partial_{x}^{2}\left(G * F_{2}\right) \Phi_{N}\right\|_{2 q}\right) \mathrm{d} \tau\right) .
\end{aligned}
$$

Now, for any function $f \in L^{1} \cap L^{\infty}, \lim _{n \rightarrow \infty}\|f\|_{L^{n}}=\|f\|_{L^{\infty}}$. Since we have that $F_{1}, F_{2} \in$ $L^{1} \cap L^{\infty}$ and $G \in W^{1,1}$, we know that $\partial_{x}^{i} G * F_{1}, \partial_{x}^{j} G * F_{2} \in L^{1} \cap L^{\infty}$ (for $i=0,1$ and $j=1,2)$. Thus, by taking the limit of (5.17) as $q \rightarrow \infty$, we get

$$
\begin{aligned}
& \left\|u \Phi_{N}\right\|_{L^{\infty}}+\left\|u_{x} \Phi_{N}\right\|_{L^{\infty}} \leq e^{C K^{2} t}\left(\left\|u_{0} \Phi_{N}\right\|_{L^{\infty}}+\left\|u_{x 0} \Phi_{N}\right\|_{L^{\infty}}\right. \\
& +\int_{0}^{t}\left(\left\|\left(G * F_{1}\right) \Phi_{N}\right\|_{L^{\infty}}+\left\|\partial_{x}\left(G * F_{2}\right) \Phi_{N}\right\|_{L^{\infty}}\right) \mathrm{d} \tau \\
& \left.+\int_{0}^{t}\left(\left\|\left(\partial_{x} G * F_{1}\right) \Phi_{N}\right\|_{L^{\infty}}+\left\|\partial_{x}^{2}\left(G * F_{2}\right) \Phi_{N}\right\|_{L^{\infty}}\right) \mathrm{d} \tau\right) .
\end{aligned}
$$

A simple calculation shows that for $\theta \in(0,1)$

$$
\Phi_{N}(x) \int_{\mathbb{R}} e^{-|x-y|} \frac{1}{\Phi_{N}(y)} \mathrm{d} y \leq \frac{4}{1-\theta}=C_{0} .
$$

Thus, for any function $f, g, h \in L^{\infty}$, we have

$$
\begin{aligned}
\left\|\Phi_{N} G * f g h\right\|_{\infty} & =\frac{1}{2} \Phi_{N}(x) \int_{\mathbb{R}} e^{-|x-y|}(f g h)(y) \mathrm{d} y \\
& \leq \frac{1}{2}\left(\Phi_{N} \int_{\mathbb{R}} e^{-|x-y|} \frac{1}{\Phi_{N}(y)}\right)\|f\|_{L^{\infty}}\|g\|_{L^{\infty}}\left\|h \Phi_{N}\right\|_{L^{\infty}} \\
& \leq\|f\|_{L^{\infty}}\|g\|_{L^{\infty}}\left\|h \Phi_{N}\right\|_{L^{\infty}} .
\end{aligned}
$$

Similarly, we have

$$
\left\|\Phi_{N} \partial_{x} G * f g h\right\|_{\infty} \leq\|f\|_{L^{\infty}}\|g\|_{L^{\infty}}\left\|h \Phi_{N}\right\|_{L^{\infty}} .
$$

Therefore, since $u, v, u_{x}, v_{x}, m, n, M \in L^{\infty}$, we get

$$
\begin{aligned}
& \left\|\left(\partial_{x}^{j} G * u M\right) \Phi_{N}\right\|_{L^{\infty}} \leq C_{0}\|M\|_{L^{\infty}}\left\|u \Phi_{N}\right\|_{L^{\infty}} \leq C_{0} K^{2}\left\|u \Phi_{N}\right\|_{L^{\infty}}, j=0,1, \\
& \left\|\left(\partial_{x}^{j} G *\left(u_{x} v m\right)\right) \Phi_{N}\right\|_{L^{\infty}} \leq C_{0}\|v m\|_{L^{\infty}}\left\|u_{x} \Phi_{N}\right\|_{L^{\infty}} \leq C_{0} K^{2}\left\|u_{x} \Phi_{N}\right\|_{L^{\infty}, j=0,1,}
\end{aligned}
$$

hence,

$$
\left\|\left(\partial_{x}^{j} G * F_{1}\right) \Phi_{N}\right\|_{L^{\infty}} \leq C_{0} K^{2}\left(\left\|u \Phi_{N}\right\|_{L^{\infty}}+\left\|u_{x} \Phi_{N}\right\|_{L^{\infty}}\right), j=0,1 .
$$

Similarly, we have

$$
\left\|\left(\partial_{x}^{j} G * u_{x} M\right) \Phi_{N}\right\|_{L^{\infty}} \leq C_{0}\|M\|_{L^{\infty}}\left\|u_{x} \Phi_{N}\right\|_{L^{\infty}} \leq C_{0} K^{2}\left\|u_{x} \Phi_{N}\right\|_{L^{\infty}, j}=0,1 .
$$

For $j=2$, noticing that $\partial_{x}^{2} G * f=G * f-f$, using the similar procedure, we have

$$
\left\|\left(\partial_{x}^{2} G * u_{x} M\right) \Phi_{N}\right\|_{L^{\infty}} \leq C_{0} K^{2}\left\|u_{x} \Phi_{N}\right\|_{L^{\infty}} .
$$


Thus, we obtain

$$
\left\|\left(\partial_{x}^{j} G * F_{2}\right) \Phi_{N}\right\|_{L^{\infty}} \leq C_{0} K^{2}\left\|u_{x} \Phi_{N}\right\|_{L^{\infty}}, j=1.2 .
$$

So, by estimates (5.18), (5.20) and (5.22) we achieve the following

$$
\begin{aligned}
& \left\|u \Phi_{N}\right\|_{L^{\infty}}+\left\|u_{x} \Phi_{N}\right\|_{L^{\infty}} \\
& \quad \leq C\left(\left\|u_{0} \Phi_{N}\right\|_{L^{\infty}}+\left\|u_{x 0} \Phi_{N}\right\|_{L^{\infty}}+\int_{0}^{t}\left(\left\|u_{0} \Phi_{N}\right\|_{L^{\infty}}+\left\|u_{x 0} \Phi_{N}\right\|_{L^{\infty}}\right) \mathrm{d} \tau\right),
\end{aligned}
$$

where $C$ is a constant depending on $C_{0}, K$ and $T$.

Multiplying the second equation of the system (5.1) by $\left(v \Phi_{N}\right)^{2 q-1} \Phi_{N}$ for $q \in N$ and integrating over the real line, then differentiating the second equation of the system (5.1) with respect to the spacial variable $x$, multiplying by $\left(v_{x} \Phi_{N}\right)^{2 q-1} \Phi_{N}$ and integrating over the real line yields, using the similar steps above, we get

$$
\begin{aligned}
& \left\|v \Phi_{N}\right\|_{L^{\infty}}+\left\|v_{x} \Phi_{N}\right\|_{L^{\infty}} \\
& \leq C\left(\left\|v_{0} \Phi_{N}\right\|_{L^{\infty}}+\left\|v_{x 0} \Phi_{N}\right\|_{L^{\infty}}+\int_{0}^{t}\left(\left\|v_{0} \Phi_{N}\right\|_{L^{\infty}}+\left\|v_{x 0} \Phi_{N}\right\|_{L^{\infty}}\right) \mathrm{d} \tau\right),
\end{aligned}
$$

Adding (5.23) and (5.24), we have

$$
\begin{aligned}
& \left\|u \Phi_{N}\right\|_{L^{\infty}}+\left\|u_{x} \Phi_{N}\right\|_{L^{\infty}}+\left\|v \Phi_{N}\right\|_{L^{\infty}}+\left\|v_{x} \Phi_{N}\right\|_{L^{\infty}} \\
& \leq C\left(\left\|u_{0} \Phi_{N}\right\|_{L^{\infty}}+\left\|u_{x 0} \Phi_{N}\right\|_{L^{\infty}}+\left\|v_{0} \Phi_{N}\right\|_{L^{\infty}}+\left\|v_{x 0} \Phi_{N}\right\|_{L^{\infty}}\right. \\
& \left.\quad+\int_{0}^{t}\left(\left\|u_{0} \Phi_{N}\right\|_{L^{\infty}}+\left\|u_{x 0} \Phi_{N}\right\|_{L^{\infty}}+\left\|v_{0} \Phi_{N}\right\|_{L^{\infty}}+\left\|v_{x 0} \Phi_{N}\right\|_{L^{\infty}}\right) \mathrm{d} \tau\right) .
\end{aligned}
$$

Hence, for any $N \in \mathbb{N}$ and any $t \in[0, T]$, we have by Gronwall's inequality that

$$
\begin{aligned}
& \left\|u \Phi_{N}\right\|_{L^{\infty}}+\left\|u_{x} \Phi_{N}\right\|_{L^{\infty}}+\left\|v \Phi_{N}\right\|_{L^{\infty}}+\left\|v_{x} \Phi_{N}\right\|_{L^{\infty}} \\
& \leq C\left(\left\|u_{0} \Phi_{N}\right\|_{L^{\infty}}+\left\|u_{x 0} \Phi_{N}\right\|_{L^{\infty}}+\left\|v_{0} \Phi_{N}\right\|_{L^{\infty}}+\left\|v_{x 0} \Phi_{N}\right\|_{L^{\infty}}\right) \\
& \leq C\left(\left\|u_{0} f_{\theta}\right\|_{L^{\infty}}+\left\|u_{x 0} f_{\theta}\right\|_{L^{\infty}}+\left\|v_{0} f_{\theta}\right\|_{L^{\infty}}+\left\|v_{x 0} f_{\theta}\right\|_{L^{\infty}}\right),
\end{aligned}
$$

with $f_{\theta}=\max \left(1, e^{\theta|x|} \mid\right)$. This concludes our proof of Theorem 5.1.

In Theorem 5.1, the exponential decay is fast decay. Next, we consider a slower decay rate which shows in the following theorem

Theorem 5.2 Assume that $s \geq 3, T>0$, and $z \in C\left([0 ; T] ; H^{s} \times H^{s}\right)$ is a solution of (1.1). If the initial data $z_{0}(x)=z(0, x)$ decays at infinity, more precisely, if there is some $\alpha \in(0,1]$ such that as $|x| \rightarrow \infty$

$$
\begin{aligned}
\left|u_{0}(x)\right| & \sim O\left((1+|x|)^{-\alpha}\right),\left|u_{0 x}(x)\right| \sim O\left((1+|x|)^{-\alpha}\right), \\
\left|v_{0}(x)\right| & \sim O\left((1+|x|)^{-\alpha}\right),\left|v_{0 x}(x)\right| \sim O\left((1+|x|)^{-\alpha}\right),
\end{aligned}
$$

then as $|x| \rightarrow \infty$, we have

$$
\begin{array}{ll}
|u(t, x)| \sim O\left((1+|x|)^{-\alpha}\right), & \left|u_{x}(t, x)\right| \sim O\left((1+|x|)^{-\alpha}\right), \\
|v(t, x)| \sim O\left((1+|x|)^{-\alpha}\right), & \left|v_{x}(t, x)\right| \sim O\left((1+|x|)^{-\alpha}\right),
\end{array}
$$

uniformly with respect to $t \in[0, T]$. 
Proof We introduce another weight function as follows:

$$
\Psi_{N}(x)=\left\{\begin{array}{l}
(1+|x|)^{\alpha},|x|<N \\
(1+|x|)^{\alpha},|x| \geq N
\end{array}\right.
$$

$N \in \mathbb{N}$ and $\theta \in(0,1)$. Observe that for all $\mathbb{N}$, we have

$$
0 \leq\left|\Psi_{N}^{\prime}\right| \leq \Psi_{N}(x), \quad \text { a.e. } x \in \mathbb{R},
$$

and there exist $C_{\alpha}$, depending only on $\alpha \in(0,1]$ such that

$$
\Psi_{N}(x) \int_{\mathbb{R}} e^{-|x-y|} \frac{1}{\Psi_{N}(y)} \mathrm{d} y \leq 3+(1+\alpha)^{2}=C_{\alpha} .
$$

Making use of $\Psi_{N}$ instead of $\Phi_{N}$ in Theorem 3.1, and then similar to the proof of Theorem 5.1. It is easy to get the desired result. Therefore, we complete the proof of the theorem 5.2.

Remark 5.1 In fact, Theorem 5.1 (resp. Theorem 5.2) tells us that the strong solution of the system (1.1) corresponding to initial data with exponential (resp. algebraical) decay at infinity will be asymptotically in the $x$-variable at infinity in its lifespan.

Acknowledgements The authors would like to thank the referees for constructive suggestions and comments. The work of Mi is partially supported by NSF of China (11671055), partially supported by NSF of Chongqing (cstc2018jcyjAX0273), partially supported by Key project of science and technology research program of Chongqing Education Commission (KJZD-K20180140).

\section{References}

1. Baouendi, M., Goulaouic, C.: Sharp estimates for analytic pseudodifferential operators and application to the Cauchy problems. J. Differ. Equ. 48, 241-268 (1983)

2. Bona, J., Smith, R.: The initial-value problem for the Korteweg-de Vries equation. Philos. Trans. R. Soc. Lond. Ser. A 278, 555-601 (1975)

3. Bressan, A., Constantin, A.: Global dissipative solutions of the Camassa-Holm equation. Anal. Appl. 5, 1-27 (2007)

4. Bressan, A., Constantin, A.: Global conservative solutions of the Camassa-Holm equation. Arch. Ration. Mech. Anal. 183, 215-239 (2007)

5. Chemin, J.: Localization in Fourier space and Navier-Stokes system, Phase Space Analysis of Partial Differential Equations. In: Proceedings, CRM series, Pisa, 53-136 (2004)

6. Constantin, A.: Finite propagation speed for the Camassa-Holm equation. J. Math. Phys. 46, 023506 (2005)

7. Constantin, A.: On the scattering problem for the Camassa-Holm equation. Proc. R. Soc. Lond. A 457, 953-970 (2001)

8. Constantin, A.: The trajectories of particles in Stokes waves. Invent. Math. 166, 23-535 (2006)

9. Constantin, A.: On the inverse spectral problem for the Camassa-Holm equation. J. Funct. Anal. 155, 352-363 (1998)

10. Constantin, A., Escher, J.: Wave breaking for nonlinear nonlocal shallow water equations. Acta Math. 181, 229-243 (1998)

11. Constantin, A., Escher, J.: Particle trajectories in solitary water waves. Bull. Am. Math. Soc. 44, 423-431 (2007)

12. Constantin, A., Escher, J.: Analyticity of periodic traveling free surface water waves with vorticity. Ann. Math. 173, 559-568 (2011)

13. Constantin, A., Gerdjikov, V., Ivanov, R.I.: Inverse scattering transform for the Camassa-Holm equation. Inverse Probl. 22, 2197-2207 (2006)

14. Constantin, A., Kappeler, T., Kolev, B., Topalov, T.: On Geodesic exponential maps of the Virasoro group. Ann. Global Anal. Geom. 31, 155-180 (2007)

15. Constantin, A., Lannes, D.: The hydro-dynamical relevance of the Camassa-Holm and DegasperisProcesi equations. Arch. Ration. Mech. Anal. 193, 165-186 (2009) 
16. Constantin, A., McKean, H.P.: A shallow water equation on the circle. Commun. Pure Appl. Math. 52, 949-982 (1999)

17. Camassa, R., Holm, D.: An integrable shallow water equation with peaked solitons. Phys. Rev. Lett. 71, 1661-1664 (1993)

18. Constantin, A., Ivanov, R., Lenells, J.: Inverse scattering transform for the Degasperis-Procesi equation. Nonlinearity 23, 2559-2575 (2010)

19. Constantin, A., Strauss, W.: Stability of peakons. Commun. Pure Appl. Math. 53, 603-610 (2000)

20. Danchin, R.: A few remarks on the Camassa-Holm equation. Differ. Integral Equ. 14, 953-988 (2001)

21. Danchin, R.: Fourier analysis methods for PDEs, Lecture Notes, 14 (2003)

22. Degasperis, A., Holm, D.D., Hone, A.N.W.: A new integrable equation with peakon solutions. Theor. Math. Phys. 133, 1461-72 (2002)

23. Degasperis, A., Procesi, M.: Asymptotic integrability. In: Degasperis, A., Gaeta, G. (eds.) Symmetry and Perturbation Theory, pp. 23-37. World Scientific, Singapore (1999)

24. Fokas, A., Fuchssteiner, B.: Symplectic structures, their Backlund transformation and hereditary symmetries. Physica D 4, 47-66 (1981)

25. Geng, X., Xue, B.: An extension of integrable peakon equations with cubic nonlinearity. Nonlinearity 22 , $1847-1856(2009)$

26. Gui, G., Liu, Y.: On the global existence and wave-breaking criteria for the two-component CamassaHolm system. J. Funct. Anal. 258, 4251-4278 (2010)

27. Grillakis, M., Shatah, J., Strauss, W.: Stability theory of solitary waves in the presence of symmetry. J. Funct. Anal. 74, 160-197 (1987)

28. Himonas, A., Mantzavinos, D.: The initial value problem for a Novikov system. J. Math. Phys. 57, 071503 (2016)

29. Henry, D.: Compactly supported solutions of the Camassa-Holm equation. J. Nonlinear Math. Phys. 12, 342-347 (2005)

30. Henry, D.: Persistence properties for a family of nonlinear partial differential equations. Nonlinear Anal. 70, 1565-1573 (2009)

31. Himonas, A., Misiolek, G.: Analyticity of the Cauchy problem for an integrable evolution equation. Math. Ann. 327, 575-584 (2003)

32. Himonas, A., Holliman, C.: The Cauchy problem for the Novikov equation. Nonlinearity 25, 449-479 (2012)

33. Himonas, A., Holliman, C.: The Cauchy problem for a generalized Camassa-Holm equation. Adv. Differ. Equ. 19, 161-200 (2014)

34. Himonas, A., Misiołek, G.: Non-uniform dependence on initial data of solutions to the Euler equations of hydrodynamics. Commun. Math. Phys. 296, 285-301 (2009)

35. Himonas, A., Misiołlek, G., Ponce, G., Zhou, Y.: Persistence properties and unique continuation of solutions of the Camassa-Holm equation. Commun. Math. Phys. 271, 511-522 (2007)

36. Holden, H., Raynaud, X.: Dissipative solutions for the Camassa-Holm equation. Discret. Contin. Dyn. Syst. 24, 1047-1112 (2009)

37. Holden, H., Raynaud, X.: Global conservative solutions of the Camassa-Holm equations-a Lagrangianpoiny of view. Commun. Partial Differ. Equ. 32, 1511-1549 (2007)

38. Holm, D.D., Staley, M.F.: Wave structure and nonlinear balances in a family of evolutionary PDEs. SIAM J. Appl. Dyn. Syst. 2, 323-380 (2003)

39. Home, A.N.W., Wang, J.P.: Integrable peakon equations with cubic nonlinearity. J. Phys. A 41, 372002 (2008)

40. Hone, W., Lundmark, H., Szmigielski, J.: Explicit multipeakon solutions of Novikov cubically nonlinear integrable Camassa-Holm type equation. Dyn. Partial Differ. Equ. 6, 253-289 (2009)

41. Hu, Q., Qiao, Z: Analyticity, Gevrey regularity and unique continuation for an integrable multi-component peakon system with an arbitrary polynomial function. arXiv:1511.03315 (2015)

42. Jiang, Z., Ni, L.: Blow-up phenomenon for the integrable Novikov equation. J. Math. Anal. Appl. 385, 551-558 (2012)

43. Kato, T., Ponce, G.: Commutator estimates and the Euler and Navier-Stokes equations. Commun. Pure Appl. Math. 41, 891-907 (1988)

44. Lai, S., Wu, Y.: The local well-posedness and existence of weak solutions for a generalized Camassa-Holm equation. J. Differ. Equ. 248, 2038-2063 (2010)

45. Lenells, J.: Traveling wave solutions of the Degasperis-Procesi equation. J. Math. Anal. Appl. 306, 72-82 (2005)

46. Li, H.: Two-component generalizations of the Novikov equation. J. Nonlinear Math. Phys. 26, 390-403 (2019) 
47. Liu, X., Yin, Z.: Local well-posedness and stability of peakons for a generalized Dullin-Gottwald-Holm equation. Nonlinear Anal. 74, 2497-2507 (2011)

48. Li, H., Liu, Q.P.: On bi-Hamiltonian structure of two-component Novikov equation. Phys. Lett. A 377, 257-281 (2013)

49. Lundmark, H., Szmigielski, J.: Multi-peakon solutions of the Degasperis-Procesi equation. Inverse Probl. 21, 1553-1570 (2005)

50. Matsuno, Y.: Multisoliton solutions of the Degasperis-Procesi equation and their peakon limit. Inverse Probl. 19, 1241-1245 (2003)

51. Misiolek, G.A.: Shallow water equation as a geodesic flow on the Bott-Virasoro group. J. Geom. Phys. 24, 203-208 (1998)

52. Ni, L., Zhou, Y.: Well-posedness and persistence properties for the Novikov equation. J. Differ. Equ. 250, 3002-3021 (2011)

53. Ni, L., Zhou, Y.: A new asymptotic behavior of solutions to the Camassa-Holm equation. Proc. Am. Math. Soc. 140, 607-614 (2012)

54. Novikov, V.: Generalization of the Camassa-Holm equation. J. Phys. A 42, 342002 (2009)

55. Taylor, M.: Partial Differential Equations III, Nonlinear Equations. Springer, Berlin (1996)

56. Tiglay, F.: The periodic Cauchy problem for Novikov equation. Int. Math. Res. Not. 2011, 4633-4648 (2011)

57. Toland, J.F.: Stokes waves. Topol Methods Nonlinear Anal. 7, 1-48 (1996)

58. Vakhnenko, V.O., Parkes, E.J.: Periodic and solitary-wave solutions of the Degasperis-Procesi equation. Chaos Solitons Fractals 20, 1059-1073 (2004)

59. Wu, X., Yin, Z.: Well-posedness and global existence for the Novikov equation. Annali Sc. Norm. Sup. Pisa. X I, 707-727 (2012)

60. Wu, X., Yin, Z.: Global weak solutions for the Novikov equation. J. Phys. A 44, 055202 (2011)

61. Xia, B., Qiao, Z.: A new two-component integrable system with peakon solutions. Proc. R. Soc. A Math. Phys. Eng. 471, 20140750 (2015)

62. Xia, B., Qiao, Z., Zhou, R.: A synthetical two-component model with Peakon solutions. Stud. Appl. Math. 135, 248-276 (2015)

63. Yan, K., Qiao, Z., Yin, Z.: Qualitative analysis for a new integrable two-component Camassa-Holm system with peakon and weak kink solutions. Commun. Math. Phys. 336, 581-617 (2015)

64. Yan, W., Li, Y., Zhang, Y.: Global existence and blow-up phenomena for the weakly dissipative Novikov equation. Nonlinear Anal. 75, 2464-2473 (2012)

Publisher's Note Springer Nature remains neutral with regard to jurisdictional claims in published maps and institutional affiliations. 